\title{
Influence of fault roughness on surface displacement: from numerical simulations to coseismic slip distributions
}

\author{
Lucile Bruhat $^{1 *}$, Yann Klinger ${ }^{1}$, Amaury Vallage ${ }^{1,2}$ and Eric M. Dunham ${ }^{3}$ \\ ${ }^{1}$ Institut de Physique du Globe de Paris, Université de Paris, CNRS UMR 7154, Paris, France \\ ${ }^{2}$ CEA, DAM, DIF, F-91297 Arpajon, France \\ ${ }^{3}$ Department of Geophysics, Stanford University, Stanford, California, USA \\ ${ }^{*}$ Now at Laboratoire de Géologie, École Normale Supérieure, PSL Research University, Paris, France
}

This is a non-peer reviewed preprint submitted to EarthArXiv.

It has been submitted on July 9, 2019 to Geophys. J. Int.

\section{SUMMARY}

Field studies characterized early on natural faults as rough, i.e. non-planar at all scales. Fault roughness induces local stress perturbations, which dramatically affect rupture behavior, resulting in slip heterogeneity. The relation between fault roughness and produced slip remains, however, a key knowledge gap in current numerical and field studies. In this study, we analyze numerical simulations of earthquake rupture to determine how roughness influences final slip profiles. Using a rupture catalog of thousands dynamic rupture simulations on non-planar fault profiles with varying roughness and background shear stress levels, we demonstrate that fault roughness affects the spectral characteristics of the resulting slip distribution. In particular, slip distributions become increasingly more self-affine, i.e. containing more short wavelength at smaller scale, with higher fault roughness, despite self-similar initial fault profiles. We also show that, at very short wavelength $(<1 \mathrm{~km})$, the fractal dimension of the slip distributions dramatically changes with 
increasing roughness, background shear stress, and rupture behavior (e.g., sub-Rayleigh vs. supershear). The existence of a critical wavelength around $1 \mathrm{~km}$, under which more short wavelength are either preserved or created, suggests the role of rupture process and dynamic effects, together with fault geometry, in resulting slip distributions. The same spectral analysis is finally performed on high-resolution coseismic slip distributions from real strike-slip earthquakes. Compared to numerical simulations, all events present slip distributions that are much more self-affine than the profiles from numerical simulations. A different critical wavelength, here around 5-6 km, appears, potentially informing about a critical asperity length. While we show here that the relation between fault roughness and produced slip distribution is much more complex than expected, this study is a first attempt at using statistical analyses of numerical simulations on rough faults to investigate observed coseismic slip distributions.

Key words: Earthquake dynamics, Rheology and friction of fault zones, Dynamics and mechanics of faulting, Mechanics, theory, and modelling. 


\section{INTRODUCTION}

While the simplest description of a fault assumes a planar surface, it has been widely recognized that the surface of natural fault is fractal, i.e., deviate from planarity in a similar way at all scales (Power \& Tullis 1991, 1995; Candela et al. 2009, 2012). This characteristic, often described as the fault roughness, has considerable effects on earthquake rupture behavior. On a rough fault, local stresses are perturbed during rupture growth, leading to complex rupture behavior. Manifestations of this complexity include rapid accelerations and decelerations of the rupture front, slip heterogeneity (Chester \& Chester 2000; Dieterich \& Smith 2009; Dunham et al. 2011b; Shi \& Day 2013), resistance to slip (Dieterich \& Smith 2009; Fang \& Dunham 2013), supershear transitions (Bruhat et al. 2016), variability in moment release (Zielke et al. 2017), in nucleation processes (Harbord et al. 2017; Tal \& Hager 2018; Tal et al. 2018; Ozawa et al. 2019), and inelastic deformation (Hirakawa \& Ma 2018). Such rupture behavior is also of particular interest to earthquake engineers when modeling building response, since rupture variability produces high frequency waves, and subsequent ground motion (Haskell 1964; Spudich \& Frazer 1984; Dunham et al. 2011b; Shi \& Day 2013). At first sight, irregularities in fault geometry provide a simple explanation for commonly observed spatial and temporal variations of fault slip (Andrews 1980). However, further studies of the influence of fault roughness on earthquake source parameter are still needed to unravel the physical mechanisms relating fault geometry to rupture behavior.

This study explores how fault roughness affects the slip distribution produced during an earthquake. Through a statistical analysis of a thousand dynamic rupture simulations on non-planar fault profiles compiled in Fang \& Dunham (2013) and Bruhat et al. (2016), we demonstrate that the fractal dimension of slip evolves with fault roughness, background shear stress, and rupture velocity. We document the spectral characteristics of the final slip distribution, especially at very short wavelength. We then perform the same spectral analyses on high-resolution coseismic slip distributions from real strike-slip earthquakes to compare them with numerical results. Through this work, we investigate whether one can infer meaningful information about fault roughness using observed slip distributions.

While the slip distribution due to a sudden loss of stress is expected to be elliptical, symmetric with respect to the center of the fault, on an idealized plane in an elastic medium (Bilby \& Eshelby 1968), analytical and numerical studies have shown for decades that the final slip profile is easily affected by changes in fault strength, in fault geometry, and in the mechanical properties of the surrounding rock (e.g. Bürgmann et al. 1994). In nature, the slip distribution after a large earthquake can vary greatly, from close to elliptical (as in Klinger et al. 2011; Choi et al. 2018) to highly irregular (as mentioned in Scholz 2019). The shape and amplitude of the slip distribution is often interpreted a posteriori as a consequence of some known property of the fault, such as the fault geometrical path or 
the rock compliance. Likewise, due to our lack of knowledge of the entire characteristics of the fault and surrounding medium, it remains very difficult to predict detailed slip distributions. Thanks to the large amount of measured slip distributions, statistical analyses, such as Manighetti et al. (2005) have tried to clarify generic features of cumulative slip; however, given a single coseismic slip distribution, it is still very challenging to determine which fault properties is responsible for its characteristics.

The relationship between fault roughness and slip also remains complex. Early on, field studies noticed differences in fault geometry with cumulative slip. Faults that have slip larger than 10-100 m appear to show lower RMS deviations from planarity than newly-created faults (Sagy et al. 2007; Sagy \& Brodsky 2009). Fault wear can occur through various mechanism, such as abrasion, grinding, grain plucking or fault splaying. While faults appear to gradually smooth with increasing slip, recent studies have emphasized that, because wear involves both smoothing and re-roughening mechanisms, leading to a slow and inefficient process, it remains difficult to constrain the degree of smoothing with slip (Brodsky et al. 2011; Shervais \& Kirkpatrick 2016). Compilations of fractal dimension of exhumed fault surfaces ranging from $50 \mu \mathrm{m}$ to $10 \mathrm{~m}$ and surface ruptures from major continental strike-slip earthquakes in Candela et al. (2012) suggest that fault geometries all share the same selfaffine characteristic with a Hurst exponent around 0.6 in the slip direction. Unlike previous work relating displacement to fault smoothing, they found no significant relationship between the roughness amplitude and cumulative slip. This would imply that fault maturity, the notion that faults inevitably smooth with cumulative slip, might still be an ambiguous process.

In the meantime, significant progress in imaging coseismic slip distributions have been made. Subpixel image correlation of combined optical and radar satellite images is now commonly used to produce maps of the 3D components of the displacement field due to an earthquake (Leprince et al. 2007; Rosu et al. 2015). Beyond the improved description of coseismic displacement, with a pixel size of the order of 1 to 10 meters, which leads to measurements along fault every 100-200 meters, these techniques allow the identification of near-field inelastic deformation features in geometrical complexities (Milliner et al. 2015; Vallage et al. 2015), that might be related to off-fault coseismic damage (Klinger et al. 2018). Studying such precise measurements might provide us a new way at relating the fault fractal dimension with the produced coseismic slip distribution. When measurements of fault roughness remain scare and onerous, one could think of that the fractal dimension of the fault would leave an imprint in the coseismic slip distribution. If such imprint exists, and is quantifiable, coseismic slip distribution would inform about the fault roughness, and by elimination, might help us to better identify the other mechanical processes affecting the final slip distribution.

In the present work, we are pursuing this strategy to study how roughness influences final slip distributions. We first analyze numerical simulations of earthquake rupture in a statistical manner to 
document the effect of roughness on slip. For that, we consider the rupture catalog described in Fang \& Dunham (2013) and Bruhat et al. (2016), which contains several thousands of rupture simulations on self-similar faults $(H=1)$ with six different values of roughness $\alpha$ and various background shear stress levels. While most numerical studies are based on the analysis of one or, at the best, a few individual ruptures, Fang \& Dunham (2013) built an ensemble of 2-D plane strain dynamic simulations on rough faults in order to allow a statistical characterization of the rupture behavior. They focused their analysis on evaluating the roughness drag, i.e. the additional shear resistance to slip due to fault roughness. Following that, Bruhat et al. (2016) documented rupture style on rough faults for both sub-Rayleigh and supershear ruptures. They showed, for instance, that, even though supershear transient are more likely on rougher faults, sustained supershear ruptures tend to occur on smoother fault segments.

In this study, we return to this ensemble database and perform various spectral analyses to document statistically how final slip profiles evolve with increasing roughness and background shear stress. We find that, for ruptures on self-similar faults, i.e. with a Hurst coefficient equal to 1 , increasing roughness leads to displacement profiles deviating from self-similarity, with Hurst coefficients down to 0.6 . We also show that at very short wavelength $(\geq 1 / \mathrm{km})$, the slope of the power spectral density dramatically changes with roughness, background shear stress, and rupture behavior (e.g., sub-Rayleigh vs. supershear). Finally, we perform the same spectral analyses on high-resolution coseismic slip distributions from real earthquakes, and compare them to our numerical results. This work constitutes a first attempt at connecting numerical simulations of rough faults to observed coseismic slip distributions.

\section{DYNAMIC RUPTURE SIMULATIONS ON SELF-SIMILAR ROUGH FAULTS}

We review here the numerical methods developed by Dunham et al. (2011a,b) and Fang \& Dunham (2013) to build a collection of 2D plane strain dynamic rupture simulations on non-planar fault profiles.

Fault roughness is commonly quantified as followed. Consider a 1D fault profile $y=h(x)$ with zero mean. This fault is defined as fractal when the power spectral density of $h(x)$ follows a power spectra law of the form:

$$
P_{h}(k) \sim|k|^{-\beta},
$$

where $\beta$ ranges from 1 to 3 . Both the fractal dimension $D$ and the Hurst coefficient $H$ relates to this exponent, such that, for a $1 \mathrm{D}$ profile $D=(5-\beta) / 2$ and $H=(\beta-1) / 2$ (Turcotte \& Huang 1995). For a self-similar fractal $\beta=3, D=1$ and $H=1$. Such a profile has root-mean-square (RMS) 
deviations from planarity $h_{\mathrm{RMS}}$ proportional to the fault length $L$ :

$$
h_{\mathrm{RMS}}=\alpha L \text {. }
$$

where $\alpha$ is the amplitude-to-wavelength ratio of roughness. Field studies show that $\alpha$ varies, in the slipparallel direction, between $10^{-3}$ to $10^{-2}$ (Power \& Tullis 1991). Depending on the study, fault roughness either designates the fractal dimension $D$, and its associated Hurst coefficient, the amplitude-towavelength ratio $\alpha$, or all of them. In this study, as we mostly consider self-similar fault profiles, the term "roughness" designates the amplitude-to-wavelength ratio $\alpha$. Any difference in Hurst coefficient will be clearly labeled as such.

Recent high-resolution measurements of surface roughness at scales below $100 \mathrm{~m}$ suggested that natural faults are not self-similar, but self-affine (Renard et al. 2006; Candela et al. 2009, 2012). Self-affine fractals present Hurst exponents below 1 (corresponding to $\beta<3$ in equation 1). While self-similar fractals have statistically similar appearance independently to the observation scale, selfaffine profiles appear smoother at larger scales. However, as discussed by Dunham et al. (2011b), no single power law spectral density has been found yet to fit both the outcrop and the map scale. Shi \& Day (2013) also showed that self-similar models have the potential to make this connection across observation scales. Likewise, over the several orders of magnitude in scale that can be currently simulated, differences between self-similar and self-affine characteristics are expected to be very small. The use of a self-similar model, prominent in recent numerical studies (Fang \& Dunham 2013; Bruhat et al. 2016; Zielke et al. 2017; Hirakawa \& Ma 2018), seemed then appropriate to test the effect of fault roughness on rupture behavior.

This study makes use of 100 randomly-generated faults profiles for six values of $\alpha$ ranging from 0.001 to 0.012 . The fault profiles are all self-similar, i.e. the Hurst coefficients of the fault traces are equal to 1. The ensemble presented in Fang \& Dunham (2013) and Bruhat et al. (2016) considered roughness up to $\alpha=0.01$, not 0.012 . In this study, we performed additional simulations to account for $\alpha=0.012$ and new values of background shear stress. Rough faults are described by a 1D profile $y=h(x)$, shown in Figure 1, embedded in an homogeneous medium, infinite in extent. The profile length is $60 \mathrm{~km}$ and the grid spacing is $\Delta x=10 \mathrm{~m}$. We set the initial stress field $\sigma_{i j}^{0}$ to be spatially uniform, described by the background shear stress $\tau_{0}=\sigma_{x y}^{0}$, the effective normal stress $\sigma_{0}=-\sigma_{y y}^{0}$, and the angle $\Psi$ between the maximum principal compressive stress and the $x$ axis. For all simulations, $\sigma_{0}=126 \mathrm{MPa}$ and $\Psi=50^{\circ}$.

We use a rate-and-state friction law, evolving toward a strongly velocity-weakening steady state strength to perform the dynamic simulations. This friction law allows the propagation of a self-healing slip pulse at low background shear stress $\tau_{0}$ (Zheng \& Rice 1998; Dunham et al. 2011b). The back- 

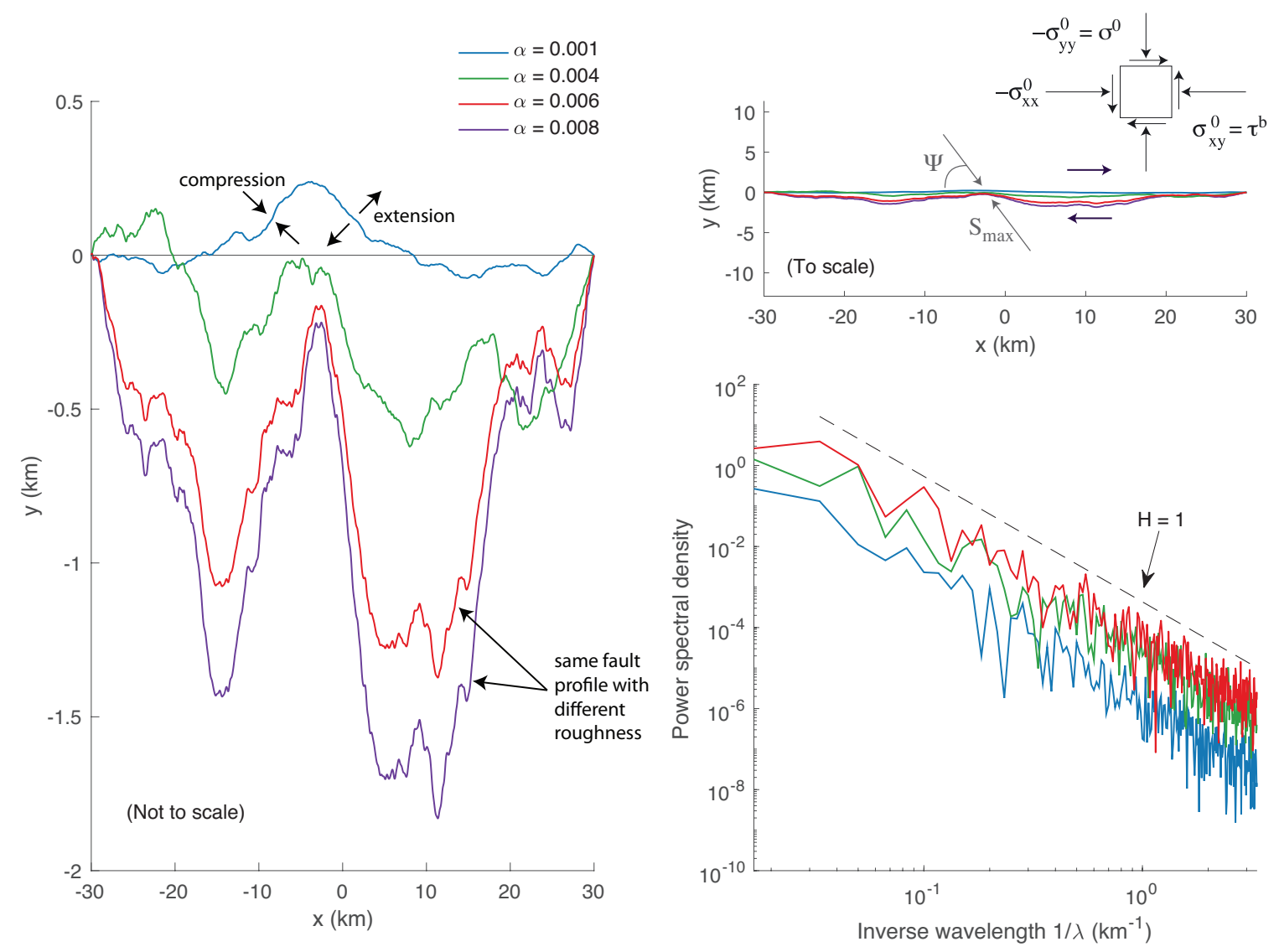

Figure 1. Left: Examples of three bandlimited self-similar faults, for four roughness values ( $\alpha=0.001,0.004$, 0.006, and 0.008), exaggerated in the y-direction, illustrating compressional and extensional bends. Top right: Plane strain model with right-lateral slip on the same faults to scale. The medium is loaded with a spatially uniform stress state, with the maximum principal stress inclined at an angle $\Psi=50^{\circ}$ to the fault. Bottom right: Corresponding power spectral density. They all follow a power spectra law of the form $|k|^{-\beta}$ where $\beta=3$ (or $H=1$ ), which defines the profiles as self-similar.

ground shear stress ranges then from $35.0 \mathrm{MPa}\left(\tau_{0} / \sigma_{0}=0.28\right)$ to $56 \mathrm{MPa}\left(\tau_{0} / \sigma_{0}=0.44\right)$. As shown by Fang \& Dunham (2013), the minimum $\tau_{0}$ at which self-sustaining propagation is possible increases with the roughness $\alpha$. A non-planar geometry will induce stress concentrations at the fault kinks. To limit these stresses and prevent fault opening, the off-fault material is characterized by a noncohesive elasto-viscoplastic rheology. Rupture nucleations are located at the point of high resolved shear-tonormal stress ratio, and triggered by applying a localized stress concentration. Each simulation generates an individual rupture, initially propagating bilaterally along a pre-defined 1D fault profile. During the earthquake lifespan, secondary ruptures of the fault can occur (examples of such complexity were displayed in Bruhat et al. (2016)). However, there is no possibility here for any fault segmentation or branching. Finally, no earthquake cycle modeling is included here, which means that the simulation 
stops once the rupture fully arrests. Further details on the modeling approach for rough faults can be found in Dunham et al. (2011a,b) and Fang \& Dunham (2013). The numerical methods are described in Dunham et al. (2011a) and Kozdon et al. (2012, 2013).

Our work focuses on a catalogue of 2D plane strain dynamic rupture simulations to analyze the rupture behavior in a statistical manner. As we vary the fault profile, the rupture will behave differently for each simulation. However, by considering a large amount of fault traces, and their associated rupture together, we are able to discriminate properties of the rupture behavior that are independent from the original fault profile. We do recognize that the recent development of 3D dynamic earthquake rupture simulations (Shi \& Day 2013; Duru \& Dunham 2016; Yao 2017; Ulrich et al. 2019) might change some of our results from 2D simulations. However, because 3D simulations remains computationally challenging, the statistical approach that we propose here has not been applied to 3D rupture simulations yet.

\section{INSIGHTS FROM NUMERICAL SIMULATIONS}

In this section, we document the influence of fault roughness on the characteristics of the slip distribution obtained from the rupture catalogue described in the previous section. For each numerical simulation, we consider the final slip distribution. We limit our analysis to well-developed ruptures, defined by having a final length larger than $10 \mathrm{~km}$. To avoid nucleation effects, we also exclude the region near the hypocenter in the following analysis.

\subsection{Effect on fractal character of the slip distribution}

As described in section 2, the fault traces produced for the rupture catalogue are all self-similar, which corresponds to Hurst coefficients close to $H=1$. For each rupture realization, we compute the power spectral density of the final slip distribution. We restrict our analysis to wavelength higher than the minimum roughness wavelength $\lambda_{\min }=0.3 \mathrm{~km}$. Due to the finite size of the domain used for the Fourier transform and the fact that most ruptures cover a substantial part of the domain, the maximum wavelength relates here to the domain size, and is here given by $1 / 60 \mathrm{~km}=0.017 \mathrm{~km}^{-1}$. Examples of final slip distribution and corresponding power spectral density are displayed in Figure 2. Like the fault profiles, the slip distributions are fractal with power spectral density described by a power spectra law $|k|^{-\beta}$ at high wavenumber. At wavelength higher than the rupture length, the spectrum becomes flat.

Using the power spectral densities of each individual slip distribution, we obtain the mean power spectral density of slip for a set of roughness $\alpha$ and background shear stress $\tau_{b}$. We then derive the 

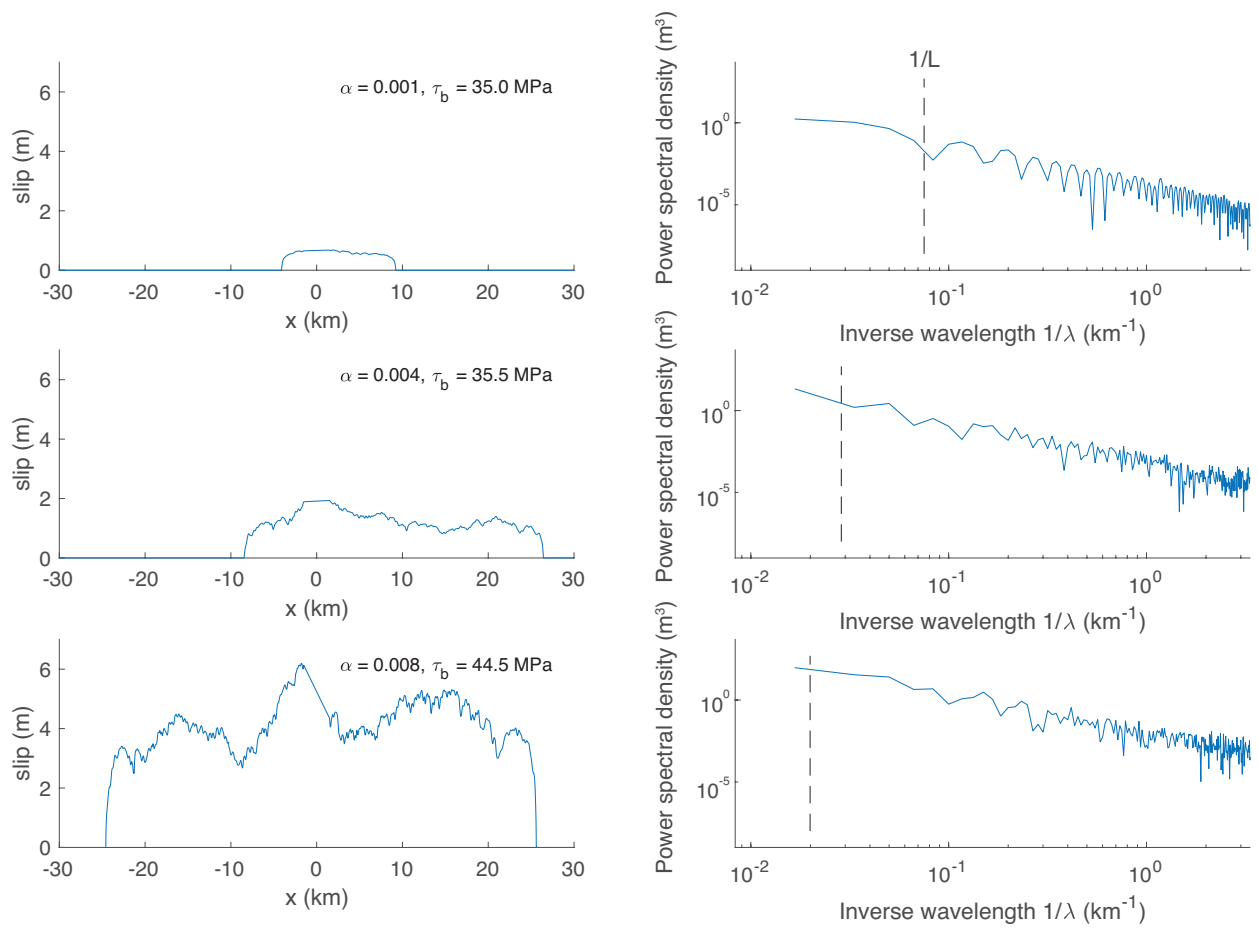

Figure 2. Examples of profile of final displacements (left panel), for three roughness values ( $\alpha=0.001,0.004$ and 0.008). Corresponding power spectral density are displayed in the right panel. At high inverse wavelength (when exceeding the rupture length $L$ ), the spectrum is described by a power spectra law $|k|^{-\beta}$. At wavelength larger than $L$, the spectrum becomes flat.

slope of the power spectral density $\beta$, and the corresponding Hurst coefficient using the relation $H=$ $(\beta-1) / 2$. Because we consider only the slip distributions of length larger than $10 \mathrm{~km}$, the slope of power spectral density is computed between $1 /(10 \mathrm{~km})$ and the minimum roughness wavelength $1 / \lambda_{\min }=1 /(0.3 \mathrm{~km})$

The evolution of the mean Hurst coefficient of the slip profiles for ruptures with varying fault roughness and background shear stress is displayed in Figure 3. At low roughness $(\alpha=0.001)$ Hurst coefficients are close to 1 . So for a very smooth fault, the slip distribution is also a self-similar fractal distribution. As the roughness increases, the range for Hurst coefficients decreases, down to 0.6 for the roughest faults $(\alpha=0.012$ ). In other words, increasing roughness, up to $\alpha=0.006$, leads to slip distribution increasingly deviating from self-similarity. Above $\alpha=0.006$, the distribution of mean Hurst coefficients seems to saturates around $0.55-0.7$. Note that this trend does not appear to relate strongly with background shear stress.

As shown in Bruhat et al. (2016), for the considered values of background shear stress, supershear transients are more likely to occur when fault roughness reaches $\alpha=0.006$. Figure 4 displays the evolution of Hurst coefficients when distinguishing sub-Rayleigh from supershear ruptures. As earlier 


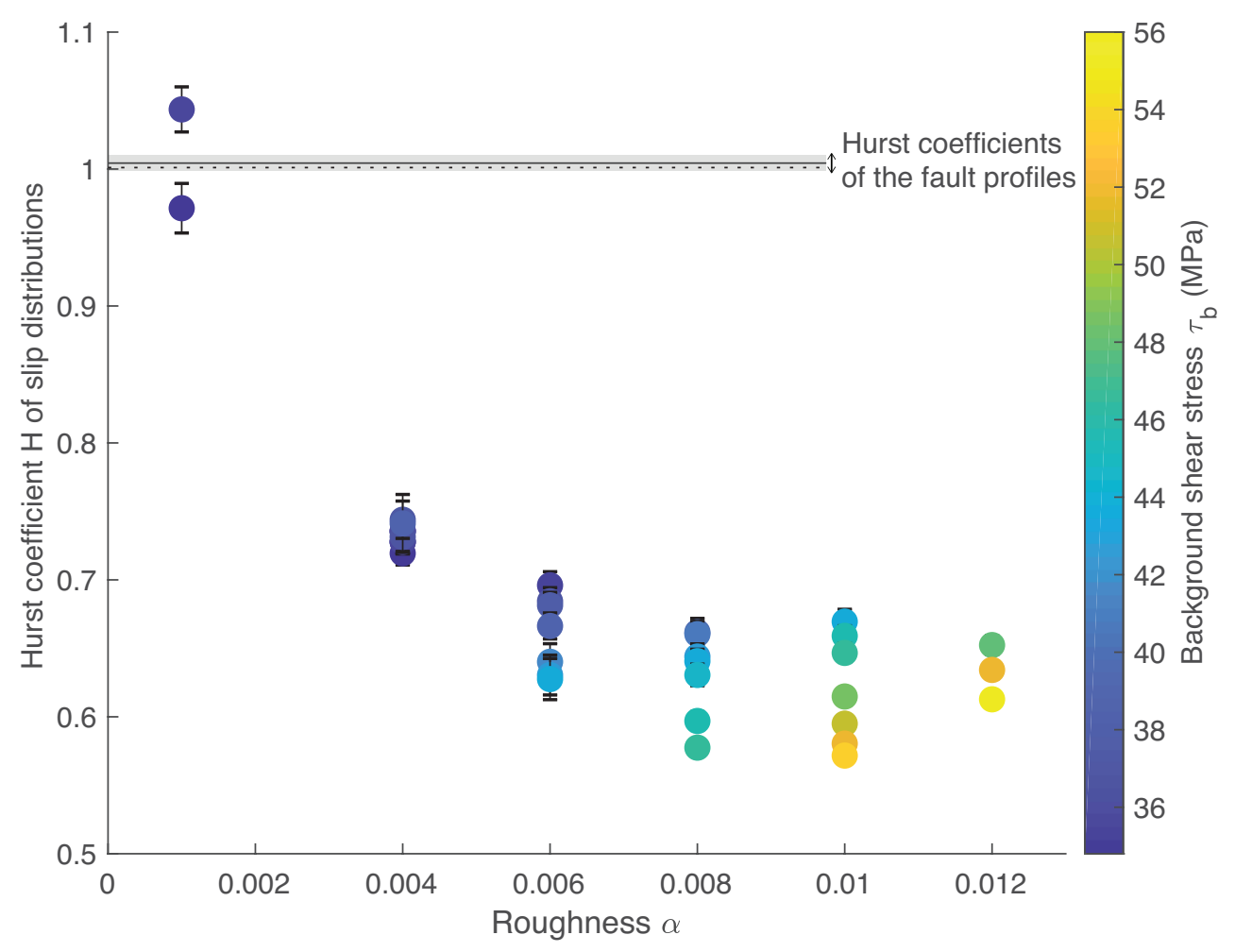

Figure 3. Evolution of the mean Hurst coefficient of final slip profiles for ruptures with varying fault roughness (x-axis) and background shear stress (colored scale). Error bars correspond to 1- $\sigma$ uncertainties. The range of Hurst coefficients of the original fault traces is also displayed. Note that, although the fault profiles were generated such that $H=1$, the uncertainty comes from the fault generation and the fitting methods. Very smooth faults ( $\alpha=0.001)$ present Hurst coefficients of slip around 1, meaning that the slip profile is selfsimilar. Increasing roughness leads to lower Hurst coefficients, down to $\sim 0.6$. Values of $H<1$ indicate that the slip distribution are not self-similar, but self-affine.

in this study, only ruptures that show the same behavior (sub-Rayleigh vs. supershear) over at least $10 \mathrm{~km}$ are considered. Figure 4 shows that only sub-Rayleigh ruptures exhibit the inverse relationship between roughness and the Hurst coefficient. On the other side, slip distributions from supershear ruptures all displays Hurst coefficients ranging from 0.55 to 0.7 . Figures 3 and 4 show that fault roughness promotes slip distribution that are more self-affine than the original fault profiles. Although the fractal character of the resulting slip distribution seems independent of background shear stress, it seems affected by the rupture velocity.

\subsection{Focus on the shorter wavelengths}

In the previous section, we estimated the slope of the power spectra density, which is computed between the minimum wavelength and $10 \mathrm{~km}$. This section first focuses on examining characteristics 

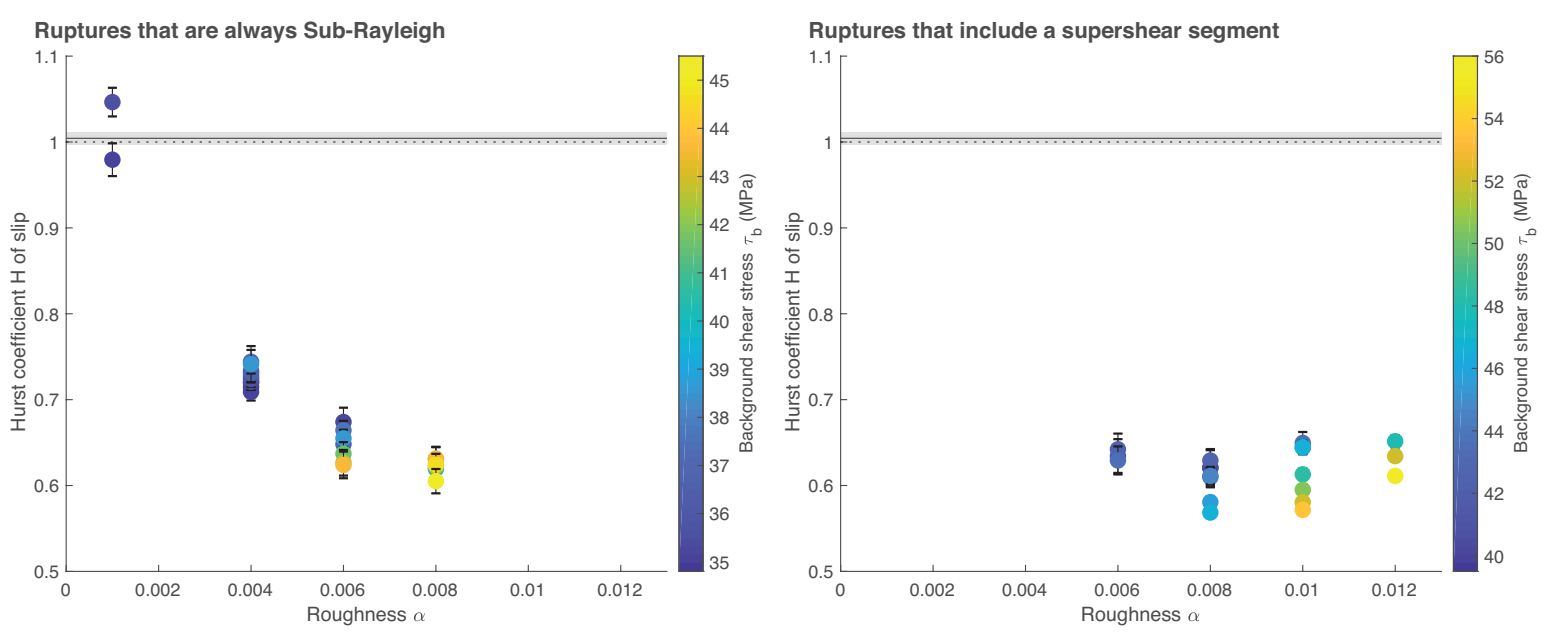

Figure 4. Distribution of Hurst coefficients when distinguishing between Sub-Rayleigh and supershear ruptures. As roughness increases, the slip distribution of Sub-Rayleigh ruptures are deviating from self-similarity. Supershear ruptures, occurring mostly on very rough faults, all show slip profiles that are self-affine, with $H \sim 0.6$.

of the power spectral densities as a function of the wavelength. Figure 5 displays the mean power spectral density of the final slip distributions, for all considered $\alpha$ and $\tau_{b}$, as a function of the inverse wavelength $1 / \lambda$, or spatial frequency. As mentioned earlier in this study, resulting spectra all decay with shorter wavelength. We still notice differences between level of roughness and background shear stress. First, the slope at short wavelength seems to increase with both $\alpha$ and $\tau_{b}$. The difference is striking between the slope observed in the spectra at $\alpha=0.001$, compared to $\alpha=0.008$. Increasing roughness leads to a greater content in short wavelength in the final slip distribution. However, this change appears to be wavelength-dependent. When focusing on the spectra for $\alpha=0.004$, we can notice that 1) the slope at very short wavelength $\left(>1 \mathrm{~km}^{-1}\right)$ differs from the one observed at longer wavelength, and that 2) when increasing the background shear stress, the slope evolves dramatically at wavelength smaller than $1 \mathrm{~km}$. Note that the value at the longest wavelength, which relates to the moment, also increases with roughness, but this increase is mostly due to increases in background shear stress, and hence stress drop.

In order to better study this behavior, we compute the power spectral density of the slip gradient distribution, instead of the slip profile. This is the slip gradient computed with respect to the horizontal distance $\mathrm{x}$. Using the gradient will help us to better distinguish changes in slope at short wavelength. Figure 6 illustrates the resulting density for $\alpha=0.004$ and $\alpha=0.006$. We observe a sharp change in slope at $1 / \lambda \sim 1 \mathrm{~km}^{-1}$. Consider the power spectral density for $\alpha=0.006$ and $\tau_{b}=35.3 \mathrm{MPa}$. The slope at which the density decreases appears to remain the same in the entire wavelength domain. Now, consider a spectrum at higher background shear stress, at wavelength shorter than $1 \mathrm{~km}$, the slope of the spectrum gradually increases, ultimately changing sign, suggesting larger amount in 

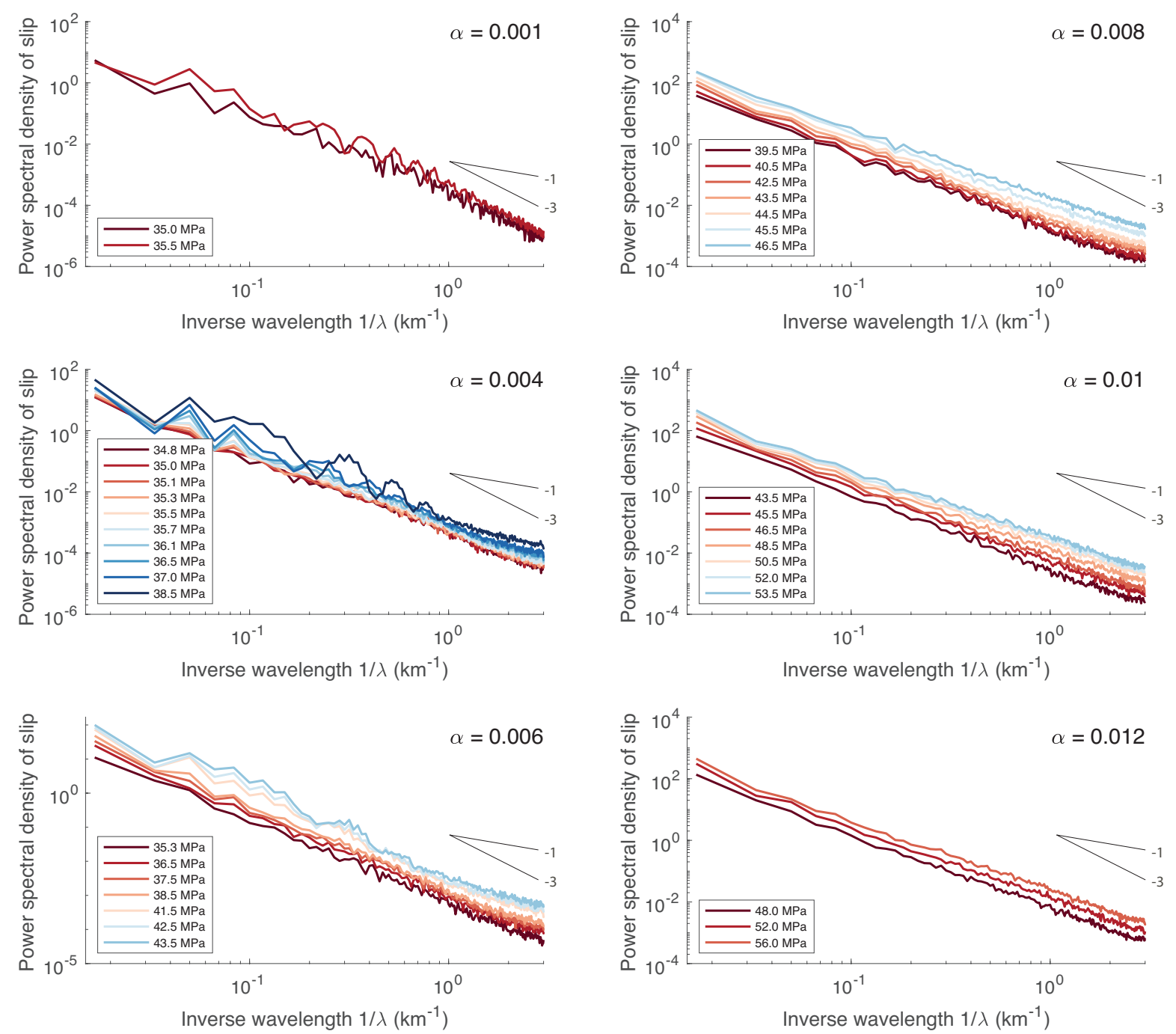

Figure 5. Mean power spectral density of final slip distributions as a function of the inverse wavelength $1 / \lambda$ and background shear stress, for roughness values from $\alpha=0.001$ to 0.012 .

shorter wavelength. Note the difference between spectrum at $\tau_{b}=35.3 \mathrm{MPa}$ and $\tau_{b}=43.5 \mathrm{MPa}$ : the slopes at $1 / \lambda>1 \mathrm{~km}^{-1}$ present opposite signs. The critical spatial frequency, at which the change in wavelength content appears also seems to vary with the roughness $\alpha$. For this particular example, increasing roughness leads to larger critical wavelength. This value appears to also depend on the background shear stress, as we notice that the sharp bend slightly moves to the left, towards longer wavelength, when increasing the background shear stress.

Following the same approach we had in Figure 3, we now compare the slope of the power spectra densities of the slip distribution, by estimating the Hurst coefficient at very short wavelength $(1 / \lambda>$ $1 \mathrm{~km}^{-1}$ ) for all level of roughness and background shear stress we consider in this study. Results are displayed in Figure 7. The slip distribution on the smoothest faults shows Hurst coefficients close to 

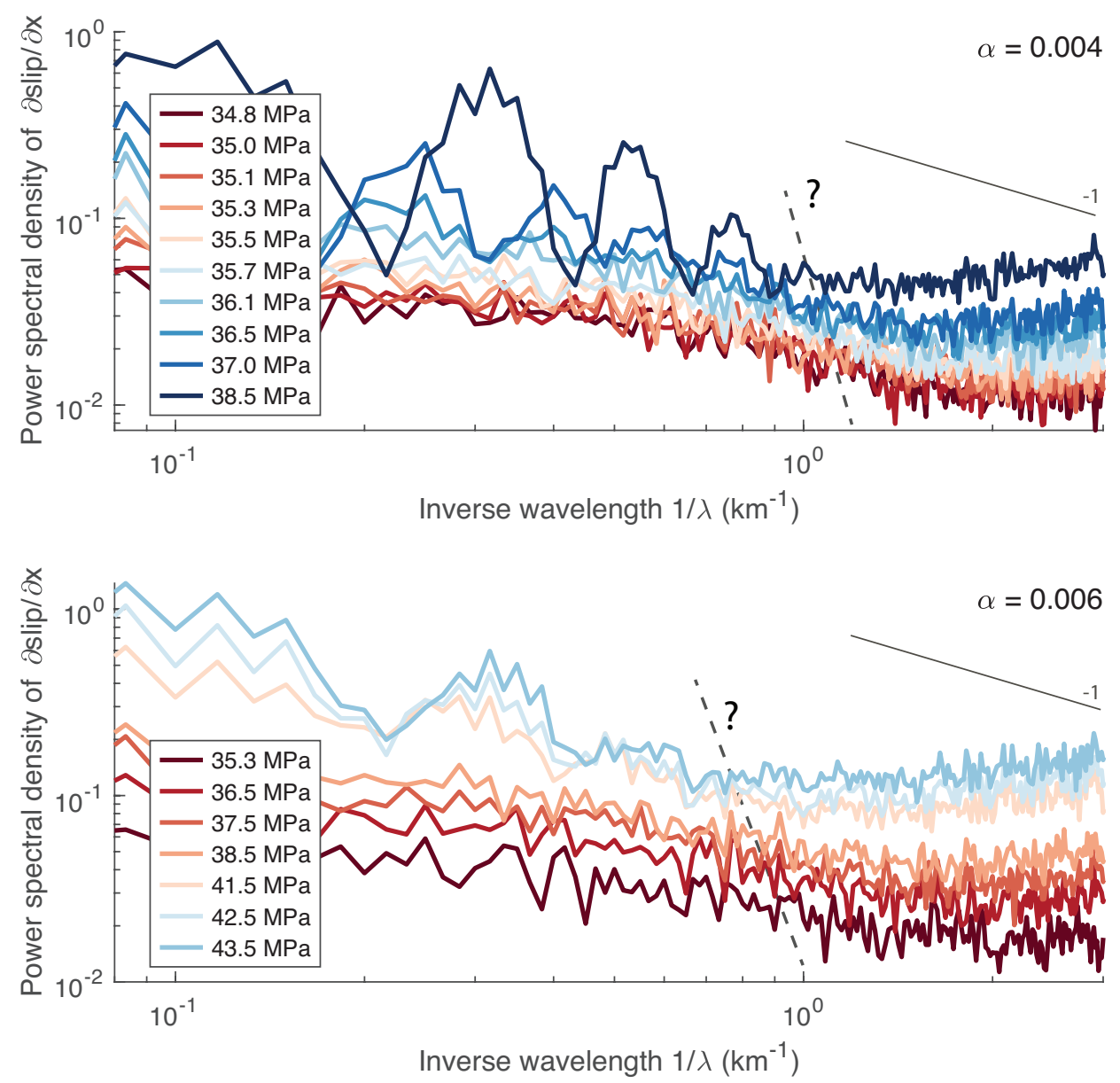

Figure 6. Mean spectra of the slip gradient of final slip distributions as a function of the inverse wavelength $1 / \lambda$ and background shear stress, for two roughness values.

1, similar to the coefficients obtained Figure 3. As the fault roughness increases, the Hurst coefficient decreases dramatically from $\sim 1$ close to almost 0.3 , up to $\alpha=0.006$. At higher roughness level, the coefficient slightly increases up to 0.6. While the results shows in Figure 3 did not seem to reveal a possible dependence with background shear stress, in this case, when we focus on the very short wavelength, the Hurst coefficient clearly decreases with increasing background shear stress for $\alpha=$ 0.004 and 0.006 (also see Figure A1). The relationship seems less obvious, even possibly reversed, for $\alpha \geq 0.006$. This might, once again, correspond to the appearance of more complex rupture behavior, such as multiple ruptures or supershear transitions (Bruhat et al. 2016).

As we notice that the change in Hurst coefficient appears around $\alpha=0.006$, we separate the ruptures that are only sub-Rayleigh from the ones that include supershear segments in Figure 8 . The distinction between the two trends in Hurst coefficients becomes clearer. As roughness increases, slip distribution from Sub-Rayleigh ruptures contains more shorter wavelengths. Increasing background shear stress emphasizes the slope reduction at short wavelength. In other words, as background shear 


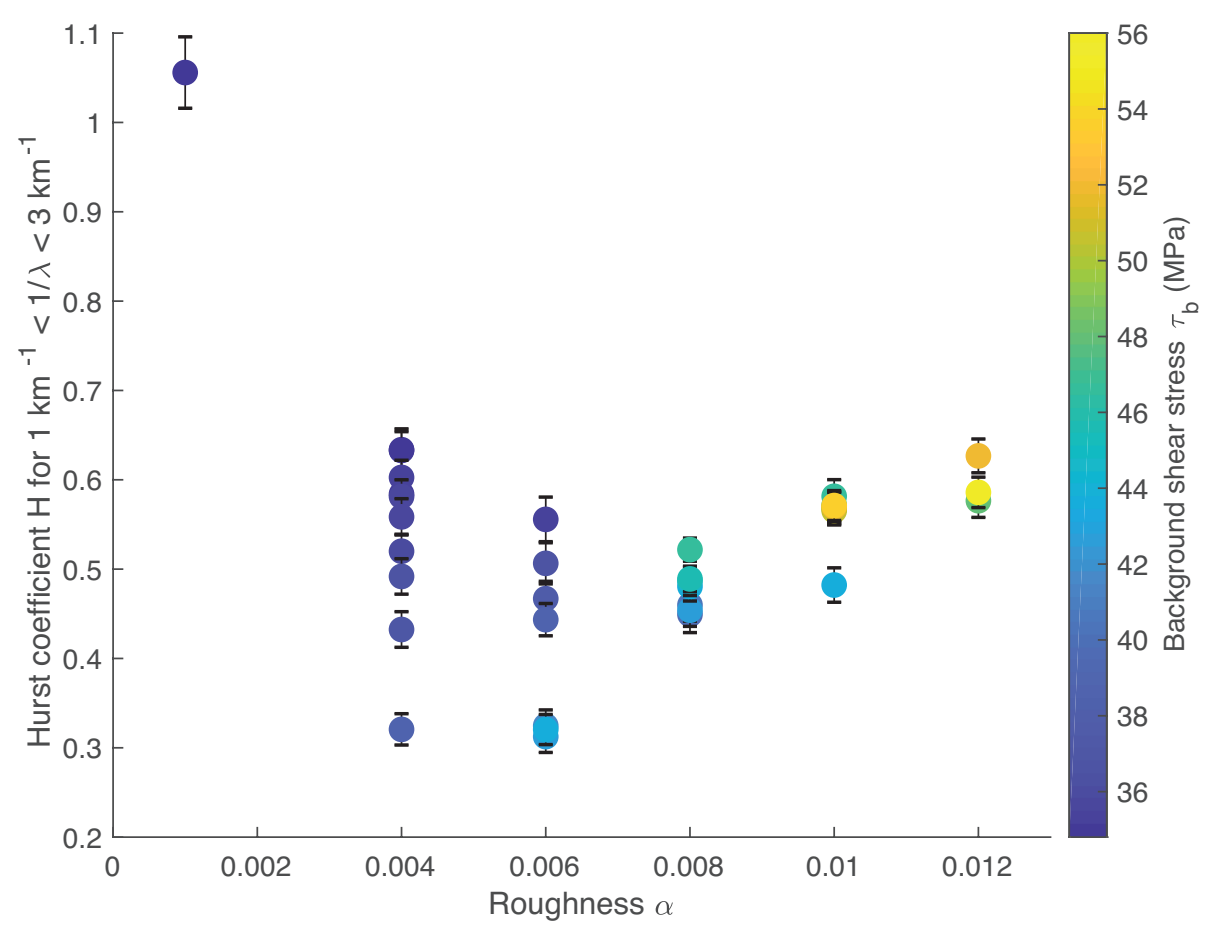

Figure 7. Evolution of the Hurst coefficient of the power spectra densities of the slip distribution, at very high spatial frequency $\left(1 / \lambda>1 \mathrm{~km}^{-1}\right)$. Error bars are for 1- $\sigma$ uncertainties. Smooth faults display Hurst coefficients close to 1 , meaning the the slip profile is self-similar. As roughness increases, the Hurst coefficients drops.
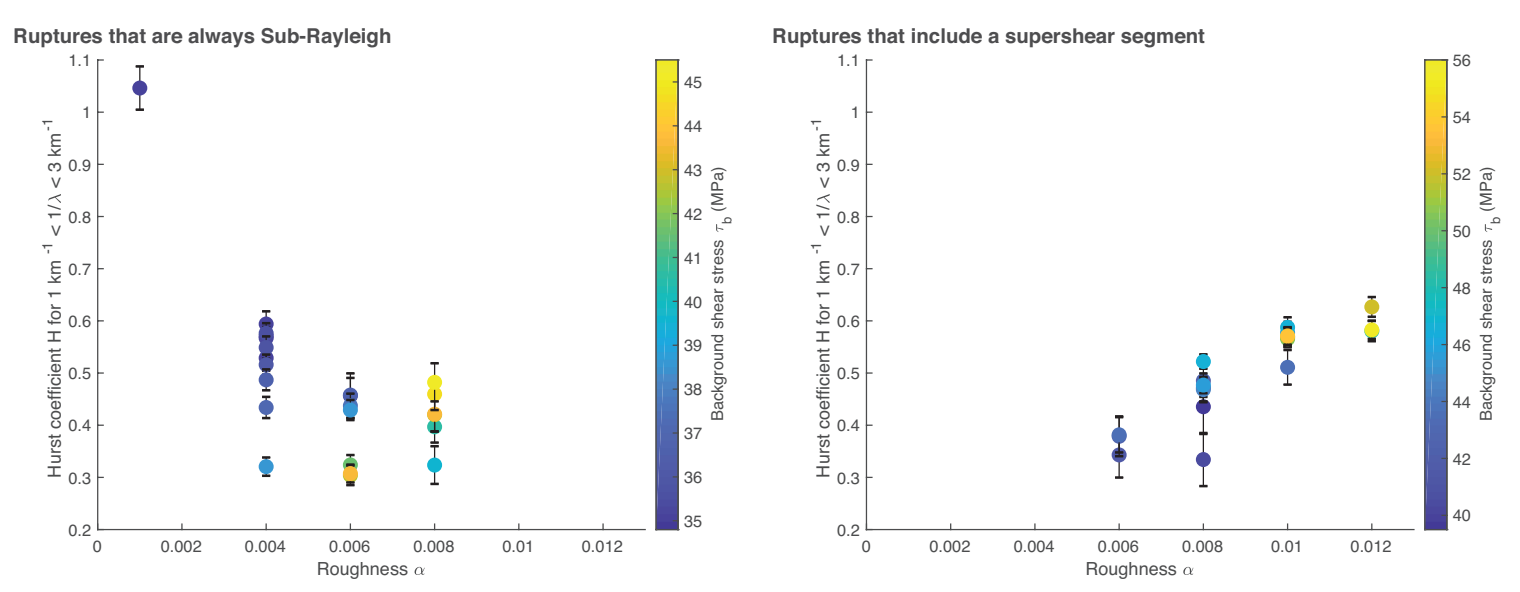

Figure 8. Distribution of the Hurst coefficient of the power spectra densities of the slip distribution at very short wavelength $\left(1 / \lambda>1 \mathrm{~km}^{-1}\right)$ when distinguishing between Sub-Rayleigh and supershear ruptures. As roughness and background shear stress increase, the Hurst coefficient decreases, meaning that the content in short wavelength in the slip distribution of Sub-Rayleigh ruptures increases. On the other side, supershear ruptures, occurring mostly on very rough faults, all show an inverse relationship between short wavelength content, and both roughness and background shear stress. 
stress increases, the final slip distribution will imprint more short wavelength content due to fault roughness. This might reflect that, as the background shear stress increases, the gradient of the resolved stress on the faults also rises, resulting in in a higher content in short wavelength in the final slip distribution. Note that at $\alpha=0.008$, although still sub-Rayleigh, the rupture becomes more complex by exhibiting secondary ruptures or rupture jumps (as shown in Bruhat et al. 2016). In this case, the final slip distribution renders the total slip history, which cannot be limited to simple single ruptures. Secondary ruptures of a region that had already ruptured might, for instance, smooth the final slip profiles, which could lead to less short wavelength, or higher Hurst coefficients. This could explain the stabilization of the range of Hurst coefficients at $\alpha=0.008$ independently of the background shear stress.

When looking at supershear ruptures, the short wavelength content in slip distributions decreases with both roughness and background shear stress. In other words, as the fault roughness or the background shear stress increases, the rupture seems less affected by the fluctuation in fault geometry, leading to smoother slip profiles. The discrepancy with the sub-Rayleigh regime might come from difference in the process zone size evolution, as it scales differently for sub-Rayleigh and supershear ruptures (Huang \& Gao 2001). However, this behavior might, once again, also result from the large rupture complexity observed at this level of roughness. As seen in Bruhat et al. (2016), multiple rupture of the same fault becomes more and more frequent leading to a final slip distribution that is not anymore representative a single rupture front, and might have smoothed due to the multiple ruptures.

\subsection{Effect on the amplitude of the slip distribution}

We finally investigate the effect of roughness on slip amplitude, by analyzing the root-mean-square slip. The root-mean-square slip of each final slip profile of length superior to $10 \mathrm{~km}$ is computed, then averaged over all the realizations. Figure 9 presents the distribution of the mean root-mean-square slip for a given set of background shear stress and roughness. As the rupture length is an important factor when considering the root-mean-square slip (Dieterich \& Smith 2009), we normalize the mean rootmean-square slip by the mean rupture length. The normalized root-mean-square slip of the ensemble is described twofold: as a function of the roughness and as a function of the background shear stress. Figure 9.a shows that the normalized mean root-mean-square slip increases linearly with the background shear stress, weakly depending of the fault roughness. This trend is similar to the equation (8) of Dieterich \& Smith (2009) that relates fault slip to length and applied stress. The normalized mean root-mean-square slip also rises with the fault roughness $\alpha$, as shown in Figure 9.b; however, this increase is mostly due to the higher background shear stress needed to nucleate on a rougher fault. 

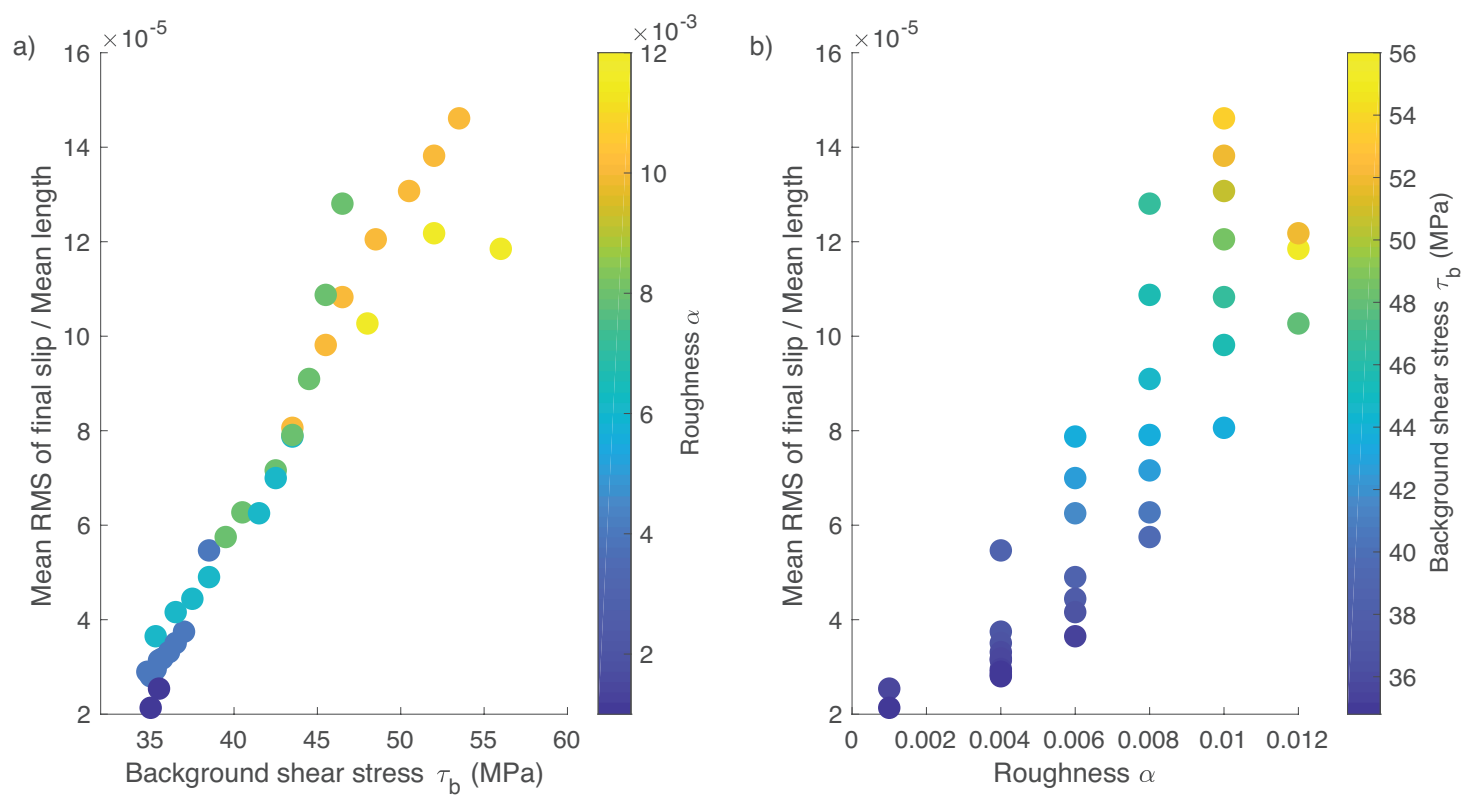

Figure 9. Distribution of the expected value of the root-mean-square slip for a given set of background shear stress and roughness. (Left) Linear relationship between the normalized mean root-mean-square of slip profiles and background shear stress, independently of the fault roughness. (Right) Normalized mean root-mean-square of slip profiles as a function of roughness. The fault roughness does not seem to have much of effect on the final slip amplitude, that is not already captured by the need for higher stress to rupture.

It seems then that the fault roughness does not have much of effect on the slip amplitude, that is not already captured by the need for higher stress to rupture.

Figure 10 illustrates these relationships for a simple bilateral sub-Rayleigh rupture. We plot the final slip profiles for two values of the background shear stress $\tau_{b}$ and for two levels of roughness $\alpha$. Increasing the background shear stress always amplifies the final slip distribution, while additional roughness impede both rupture propagation, i.e. final length, and slip amplitude. This last result is similar to conclusions by Dieterich \& Smith (2009) and Fang \& Dunham (2013). Both studies showed that roughness induces an additional shear resistance to slip proportional to $\alpha^{2}$. At fixed background shear stress, as the geometrical drag increases, the fraction of shear stress allowed to resistance to friction, and thus slip, decreases. Figure 9 and 10 showed that the slip amplitude relates mostly to background shear stress, and not as much to fault roughness. This relation is close to linear between the slip mean root-mean-square and the background shear stress. 

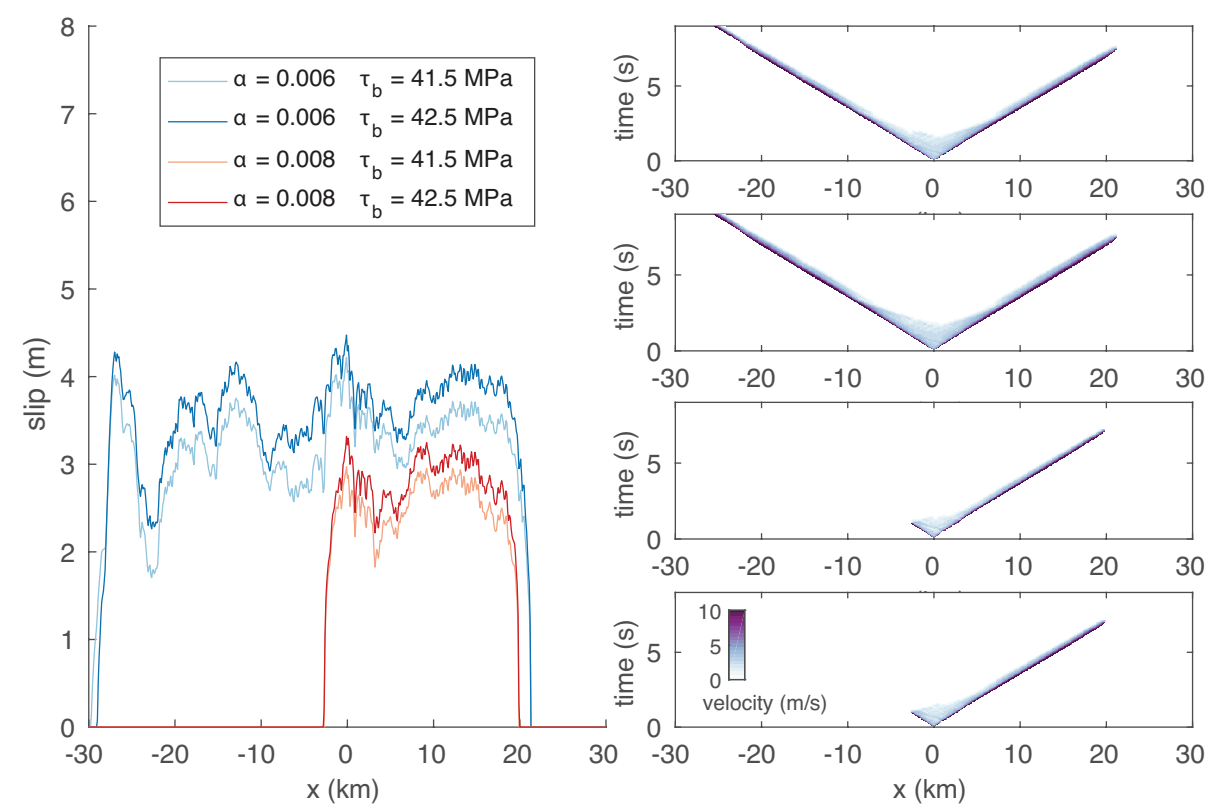

Figure 10. Influence of roughness and background shear stress on slip characteristics with $\alpha=0.006$ and 0.008 . Increasing the background shear stress always amplifies the final slip distribution, while additional roughness impede both rupture propagation, i.e. final length, and slip amplitude.

\section{COMPARISON WITH REAL STRIKE-SLIP DISTRIBUTIONS}

In this section, we compare the results from our numerical simulations with coseismic displacement profiles from large strike-slip earthquakes: the $1992 \mathrm{M}_{w}=7.3$ Landers, the $1999 \mathrm{M}_{w}=7.1$ Hector Mine, the $2013 \mathrm{M}_{w} 7.7$ Balochistan, and the $2016 \mathrm{M}_{w} 7.8$ Kaikoura earthquakes. These events were chosen because they all occurred on well-defined strike-slip faults with high-resolution measurements of surface coseismic displacements, which would constitute an appropriate analog to our 2D plane strain dynamic rupture simulations. For each coseismic slip profiles, we will follow the same analysis done with the numerical simulations by computing the power spectral density to determine Hurst coefficients and investigate its behavior at short wavelengths.

We take advantage of published maps of horizontal displacements due to large earthquakes, resulting from the recent progress in the field of image correlation of combined optical satellite images (Leprince et al. 2007; Rosu et al. 2015). We use high-resolution along-strike coseismic slip profiles of the $1992 \mathrm{M}_{w}=7.3$ Landers, and $1999 \mathrm{M}_{w}=7.1$ Hector Mine earthquakes from Milliner et al. (2016). Using subpixel correlation of satellite images, they produce profiles of right-lateral displacement every $\sim 140 \mathrm{~m}$, over $60 \mathrm{~km}$ along the rupture profiles. Likewise, Vallage et al. (2015) combined optical satellite images to produce high-resolution maps of fault-parallel and fault-normal displacement due to the $2013 \mathrm{M}_{w}$ 7.7 Balochistan earthquake in Pakistan. In this study, we consider the resulting profile of fault-parallel slip derived from far-field measurements, sampled every $100 \mathrm{~m}$ for a $200 \mathrm{~km}$-long 

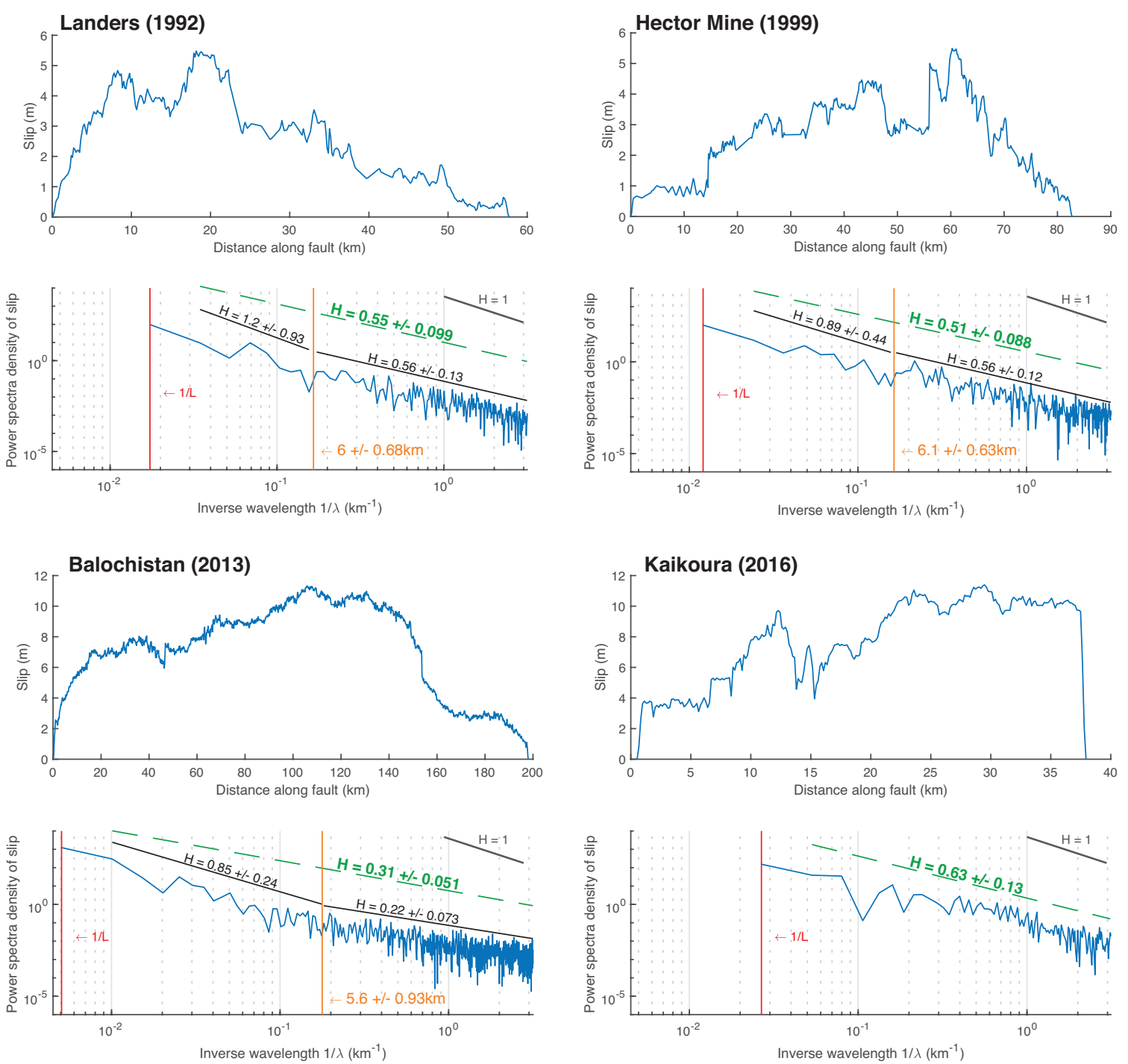

Figure 11. Coseismic slip profiles and associated power spectral densities of four large strike-slip earthquakes: the $1992 \mathrm{M}_{w}=7.3$ Landers, the $1999 \mathrm{M}_{w}=7.1$ Hector Mine, the $2013 \mathrm{M}_{w} 7.7$ Balochistan, and the $2016 \mathrm{M}_{w}$ 7.8 Kaikoura events (Milliner et al. 2016; Vallage et al. 2015; Klinger et al. 2018). Hurst coefficients $H$ are computed for all events. When the behavior at short wavelength seems to vary, we estimate the wavelength at which the change occurs and the Hurst coefficients on its both sides.

rupture. Finally, we use the fault-parallel slip distribution at every $100 \mathrm{~m}$ produced by Klinger et al. (2018) for the $2016 \mathrm{M}_{w} 7.8$ Kaikoura earthquake in New Zealand. When the earthquake involves ruptures on multiple segments, the shown slip distribution is the one following the main rupture path. In order to compare all profiles adequately in the following spectral analysis, we interpolate them to obtain one measurement every 150 m, i.e., around 6 points per km. Figure 11 displays the coseismic fault-parallel displacement distribution used in this study. For reference, we will also make use of the slip profile produced by the $2001 \mathrm{M}_{w} 7.8$ Kunlun earthquake, displayed Figure A2 (Klinger 

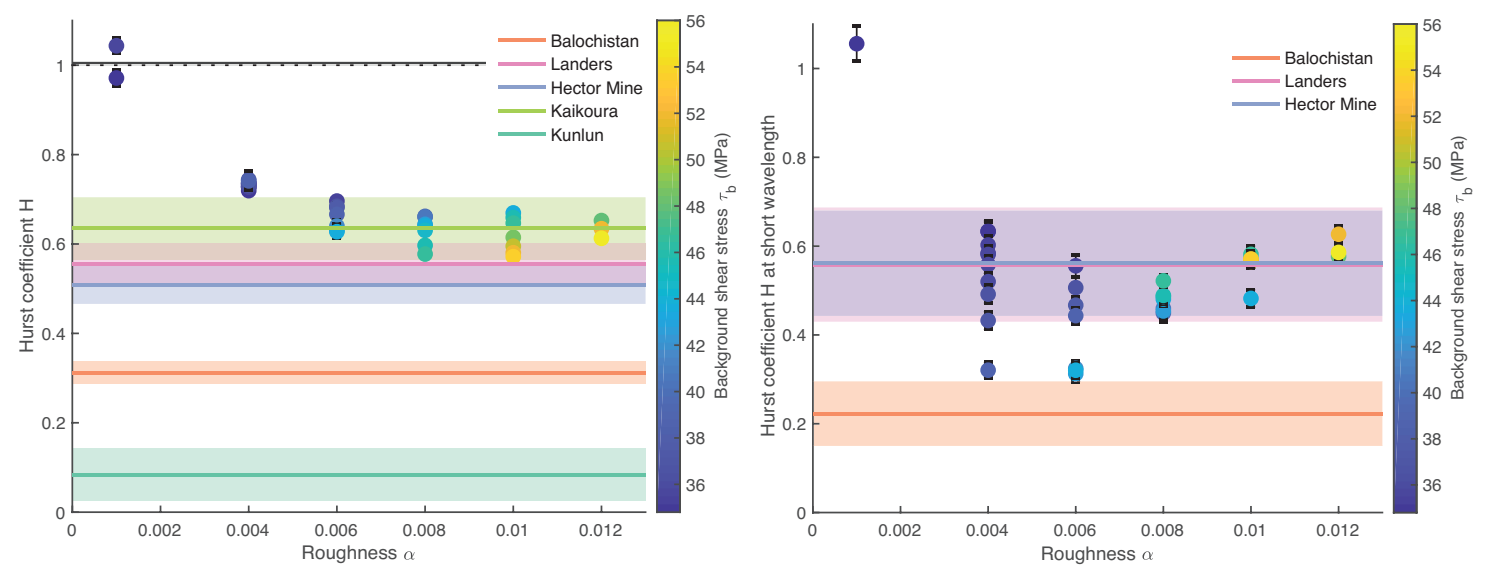

Figure 12. Comparison between the statistical analysis of mean Hurst coefficients from the numerically simulated slip profiles, lead in the previous section (Figures 2 and 7), with inferred Hurst coefficients from observed coseismic distributions.

2005). Note that compared to the other profiles, the resolution of this slip profile is inferior (up to one measurement per $\mathrm{km})$.

Using the coseismic slip distributions, we follow the same procedure we developed with the numerically-generated slip profiles. We compute the power spectral density of each distributions, then derive the Hurst coefficient for wavelengths between the rupture length and the minimum wavelength considered here, i.e. $150 \mathrm{~m}$. Resulting power spectral density are presented in Figure 11. Obtained Hurst coefficients range from 0.31 to 0.63 , indicating that the final slip distribution are all self-affine, far from self-similarity. We notice that there seems to be, once again, a decrease in slope at shorter wavelengths. For instance the power spectral density for the Balochistan earthquake appears to flatten at wavelength shorter than $5 \mathrm{~km}$.

We estimate the location of this kink using the following procedure. For a given wavelength, we compute the slopes of the spectrum on its right (at higher frequency) and left (at lower frequency) side. This procedure is then repeated for all the wavelength range considered. We obtain curves of the evolution of the slope of the low and short wavelength ends. A kink, or change in slope, will then appear as the minimum of the difference between these two slopes. For the Landers, Hector Mine and Balochistan event, this kink seems to be located around $6 \mathrm{~km}$. The procedure could not find a kink in the power spectral density for the Kaikoura earthquake. For both the Landers and Hector Mine earthquakes, the Hurst coefficients at shorter wavelength have similar values, around 0.56, compared to the ones computed over the entire wavelength domain. On the other side, the power spectral density of the Balochistan event shows lower value of Hurst coefficient at shorter wavelength, indicating a greater level in shorter wavelengths.

We now compare our results from the numerical simulations, obtained in the previous section, with 
values of Hurst coefficients that we computed from real coseismic displacement profiles. Figure 12 illustrates the distribution of the mean Hurst coefficient of numerically-generated slip profiles over the entire wavelength domain and at very short wavelength $\left(1 / \lambda>1 \mathrm{~km}^{-1}\right)$ as there are presented in Figures 3 and 7. We superimpose the computed values of Hurst coefficients from all considered earthquakes. Due to the lack of short wavelength, or to a different behavior at short wavelength, we only plot results from the Landers, the Hector Mine and the Balochistan quakes on the figure that displays Hurst coefficients computed at short wavelength. We first notice that, while the range of Hurst coefficients of the slip distributions covered by the numerical simulations range from 1 to 0.55 , the Hurst coefficients inferred from the coseismic slip profiles all lie below 0.65 . The slip distributions from the Landers, the Hector Mine and the Kaikoura earthquakes present Hurst coefficients around 0.5-0.6, close to range of the numerical simulations, but still difficult to distinguish due to the high uncertainty. On the other hand, Hurst coefficients computed from the slip profiles of the Balochistan and Kunlun earthquakes range between 0 and 0.3 , setting them away from the levels observed in numerical simulations. When comparing the slope of slip spectra at short wavelength, there is a similar agreement between numerical simulation and observed slip distributions. The Hurst coefficient obtained for the Landers and Hector Mine earthquakes lies within the same range than the numerical simulations; however, the large uncertainties prevent from drawing any conclusions about the fault roughness. The Balochistan event presents once again level of short wavelength higher than any simulations.

\section{DISCUSSION}

In this study, we investigated the characteristics of slip distributions generated on rough faults. The overarching goal of this approach is to evaluate if we can deduce information about the fault roughness and the rupture processes in observed coseismic displacement profiles. We first documented the spectral characteristics of the slip distribution with respect of the roughness and the background shear stress. We showed that, even though all profiles were generated on self-similar profiles, the slip profiles become increasingly more self-affine with higher fault roughness $\alpha$ (Figure 3 ). There is no obvious agreement between the fractal dimension of the fault profile, and the one of the resulting slip distributions. This is unexpected, because slip perturbations are expected to have the same statistical properties than the local geometry, as shown by Dunham et al. (2011b), at least in the first order static analysis. Differences might be due to rupture process and dynamic effects which, together with fault geometry, either creates more short wavelength slip fluctuations, or suppress long wavelength slip, leading to a self-affine distributions. When separating sub-Rayleigh from supershear ruptures, we noticed that the deviation from self-similarity grows linearly with the fault roughness. Slip distributions from supershear ruptures are systematically self-affine, with Hurst coefficients around 0.6 (Figure 4). We finally 


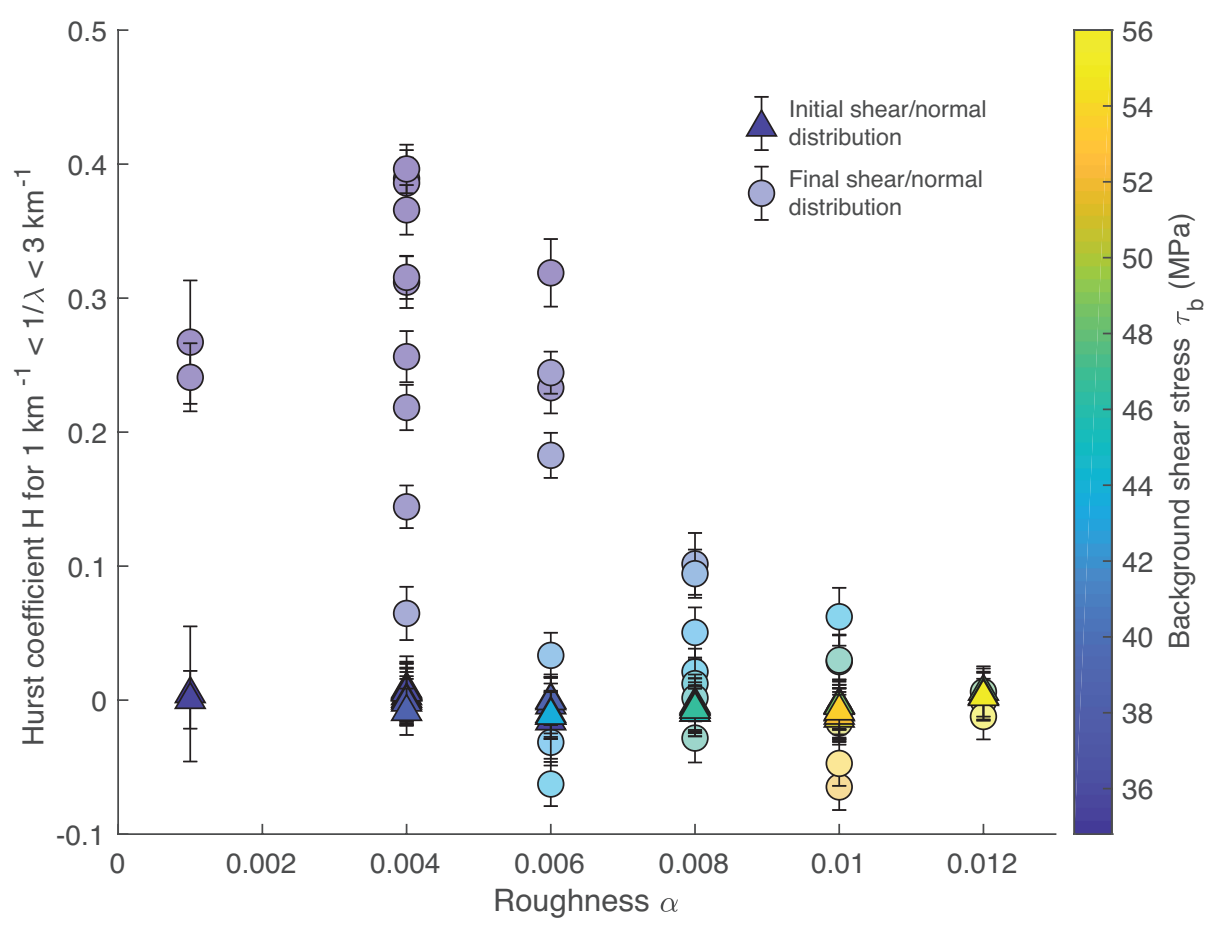

Figure 13. Distribution of the Hurst coefficient of the power spectra densities of the initial (triangles) and final (circles) shear-to-normal distribution at very short wavelength $\left(1 / \lambda>1 \mathrm{~km}^{-1}\right)$ as a function of roughness and background shear stress.

showed that the amplitude of the slip profile increases linearly with the background shear stress. The fault roughness does not seem to have such an effect, that is not already captured by the need for higher background shear stress to initially rupture (Figure 9).

Results from numerical simulations revealed that roughness and background shear stress affect the fractal dimension of the produced slip distribution. This control might, however, be affected by the set of parameters we choose to characterize the elasto-viscoplastic rheology of the off-fault material. We test this idea by running new sets of simulation for different values of plastic dilatancy $\beta$ and DruckerPrager viscosity $\eta$ in the medium surrounding the fault (see details about the tested parameters in Appendix A). Figure A1 shows that there are few changes between the Hurst coefficients obtained for the original simulations and the ones obtained with different off-fault characteristics. At the least, the few changes are not capable to explain the large variation in Hurst coefficients with roughness and background shear stress we observed in the earliest sections. Thus, the deviation from self-similarity that can be observed in slip distributions do not seem to be related to the material properties of the surrounding material .

When looking at individual sets of power spectra density, we realized that the slope, at which the density decreases, also varies with the wavelength range. We showed that there seemed to be a critical 


\section{Bruhat, et al.}

wavelength, (around $1 \mathrm{~km}$ in our simulations) above which the content in short wavelength varies with roughness and background shear stress (Figures 5 and 6). Slip profiles from Sub-Rayleigh ruptures show clearly greater content in short wavelength (i.e. lower Hurst coefficient) with increasing roughness and background shear stress. Differently, the Hurst coefficient of supershear slip profiles increases with roughness (Figure 8). For such a simple 2D numerical set-up, the existence of a critical wavelength is puzzling. As it appears clearly in the power spectral density of the slip gradient (Figure 6), we first investigate whether the initial shear-to-normal stress distribution can explain the slip variability. We perform the same spectral analysis for the initial and final shear-to-normal stress distribution, and compute the Hurst coefficient at short wavelength. Figure 13 presents the Hurst coefficient evolution with roughness and background shear stress, while the spectral power density are given in Figure A3. The initial shear-to-normal distributions all have Hurst coefficient around 0. Neither the roughness or the background shear stress seems to affect the Hurst coefficients. Note that this does not apply for the final shear-to-normal distributions whom Hurst coefficients greatly differ from the ones of the initial distributions and varies with roughness and background shear stress. Were the numerical simulations on rough faults able to do earthquake cycles, the characteristics of the shear-to-normal distribution would change dramatically between two events. The statistical characteristics of the slip distribution after multiple ruptures on a single fault might greatly vary from the slip pattern of the single ruptures we are investigating in this study. While beyond the scope of this work, this issue merits further study.

To explain the presence of a critical length scale, we also investigate whether there is any correlation between slip gradient, the slope $m(x)=\partial h / \partial x$ and rupture velocity. Unfortunately, correlations displayed Figure A4 show that no correlation appear between the slip gradient and the fault slope. Only the correlation between slope and rupture velocity, already observed in Fang \& Dunham (2013) emerges here. Finally, given that the ruptures are all self-healing pulse, the length of the pulse might play a role in this critical length scale. Differences in the Hurst coefficient for sub-Rayleigh vs. supershear ruptures suggest a control from the process zone, and subsequently the pulse width. Coincidentally, from the couple of examples given in Bruhat et al. (2016), the pulse width seems to be also around $1 \mathrm{~km}$. Further work is definitely required from the numerical side to confirm the existence of this critical wavelength, estimate it origin, and determine whether it is fully controlled by the fault roughness.

When comparing with measured coseismic slip, the Hurst coefficients obtained from numerical simulation most often overestimate the ones observed in real slip distributions. Only the Landers and the Hector Mine ruptures present Hurst coefficients of the same range than the synthetics profiles. However, the large uncertainties and the complexity revealed in the earlier analysis of the numerical simulations make it difficult, for now, to relate the spectral signature of real earthquake to fault rough- 
ness. Explanations for this negative results are multiple. First, we use a simplified fault model that does not consider other possible controls on slip heterogeneity, such as previous slip history, afterslip, poroelastic effects (Hirakawa \& Ma 2018), and most importantly, re-roughening mechanisms, such as segmentation or branching. Although the first processes would likely smooth out short wavelength features since there are associated with relaxation of stress concentrations, re-roughening mechanisms, which play important roles in fault evolution, are plausible causes for slip profiles with low Hurst coefficients. Moreover, while we set aside the Kunlun earthquake because of the lack of short wavelength, the corresponding slip distribution has a very different spectral content, with Hurst coefficient close to zero (Figure A2). Unlike the other earthquakes, this event ruptured permafrost (Klinger 2005), which acts brittle and offers little chance for damping or distributed deformation in the surrounding environment. This could result in a slip distribution with higher short wavelength content. Finally, our model ignores 3D effects, which might affect the resulting slip distribution at the surface. Although dynamic ruptures on 2D rough faults are currently developed (Duru \& Dunham 2016; Yao 2017; Williams et al. 2018), the computational cost is still too high to produce ruptures catalogues and start statistical analysis of rupture complexity.

An obvious explanation of the observed discrepancy between numerical modeling and recorded coseismic slip might originate from the assumption of self-similarity of the fault geometry. All the synthetic fault traces were generated as self-similar faults, i.e. with a Hurst coefficient of 1. Self-similar faults are commonly used in numerical simulations since they have the power to connect roughness measurements across all scale, from the outcrop to the map. The trade-off in Hurst coefficients observed in this study raises questions about using self-similar faults in the first place. Future work might consider self-affine faults as a starting point, exploring whether it would affect rupture and slip behavior as much.

A notable difference between the synthetic and observed slip distributions is the presence of a critical wavelength under which the power spectral density flattens. While this critical wavelength always occurred at $\lambda<1 \mathrm{~km}$ in the numerical simulations, it occurs around 5-6 km for the Landers, Hector Mine and Balochistan events. On the other hand, it remains difficult to see any change in slope in the power spectral density for the Kaikoura earthquake. Taken at face value, we cannot make any connection between the critical wavelength noticed in synthetic slip profiles and the one inferred for the Landers, Hector Mine and Balochistan earthquakes. The latter might informs about a segmentation, or seismogenic length, as suggested in Klinger (2010). It could also simply reveal a critical asperity size that would resist abrasional wear and fracturing. This hypothesis was already mentioned in Milliner et al. (2015) when analyzing the Landers earthquake, where the authors actually predicted a "a second roll-off or "whitening at higher wave numbers". Unfortunately, the authors did not seem to detect the 


\section{Bruhat, et al.}

critical wavelength we found with study, possibly due to the fact that there were analyzing only one rupture.

Our study started with the idea that geometrical complexity such as fault roughness possibly leaves an imprint in the produced slip distribution, and that if this signature is somewhat quantifiable, we would be able to infer properties of fault roughness from past slip distributions. Using dynamic modeling of earthquakes on rough faults, we showed that the connection between fault roughness and the spectral content of the slip distribution is much more complex, as it might depend on the initial fault geometry and the rupture behavior. While rupture behavior and dynamic effects might explain the complexity seen in numerical modeling, geometric complexities, like branches or segmentation, and wear processes, might play an additional role in real Earth. We hope, however, that the current development of high-resolution measurements of coseismic slip distribution will help enlightening the relationship between fault roughness and surface displacement.

\section{ACKNOWLEDGMENTS}

This work was supported by the ANR Geosmec ANR-12-BS06-0016. Simulations were done using the finite difference code FDMAP. Code and input files are available at https://bitbucket.org/ericmdunham/fdmap.

\section{REFERENCES}

Andrews, D. J., 1980. A stochastic fault model: 1. Static case, Journal of Geophysical Research, 85(B7), 3867.

Bilby, B. A. \& Eshelby, J. D., 1968. Dislocations and the theory of fracture, in Fracture, an advanced treatise, pp. 99-182, Academic Press New York and London.

Brodsky, E. E., Gilchrist, J. J., Sagy, A., \& Collettini, C., 2011. Faults smooth gradually as a function of slip, Earth and Planetary Science Letters, 302(1-2), 185-193.

Bruhat, L., Fang, Z., \& Dunham, E. M., 2016. Rupture complexity and the supershear transition on rough faults, Journal of Geophysical Research: Solid Earth, 121(1), 210-224.

Bürgmann, R., Pollard, D. D., \& Martel, S. J., 1994. Slip distributions on faults: effects of stress gradients, inelastic deformation, heterogeneous host-rock stiffness, and fault interaction, Journal of Structural Geology, 16(12), 1675-1690.

Candela, T., Renard, F., Bouchon, M., Brouste, A., Marsan, D., Schmittbuhl, J., \& Voisin, C., 2009. Characterization of fault roughness at various scales: Implications of three-dimensional high resolution topography measurements, Pure and Applied Geophysics, 166(10-11), 1817-1851.

Candela, T., Renard, F., Klinger, Y., Mair, K., Schmittbuhl, J., \& Brodsky, E. E., 2012. Roughness of fault surfaces over nine decades of length scales, Journal of Geophysical Research: Solid Earth, 117(B8).

Chester, F. M. \& Chester, J. S., 2000. Stress and deformation along wavy frictional faults, Journal of Geophysical Research, 105(B10), 23421-23430. 
Choi, J.-H., Klinger, Y., Ferry, M., Ritz, J.-F., Kurtz, R., Rizza, M., Bollinger, L., Davaasambuu, B., TsendAyush, N., \& Demberel, S., 2018. Geologic Inheritance and Earthquake Rupture Processes: The 1905 M 8 Tsetserleg-Bulnay Strike-Slip Earthquake Sequence, Mongolia, Journal of Geophysical Research: Solid Earth, 123(2), 1925-1953.

Dieterich, J. H. \& Smith, D. E., 2009. Nonplanar Faults: Mechanics of Slip and Off-fault Damage, Pure and Applied Geophysics, 166(10-11), 1799-1815.

Dunham, E. M., Belanger, D., Cong, L., \& Kozdon, J. E., 2011a. Earthquake Ruptures with Strongly RateWeakening Friction and Off-Fault Plasticity, Part 1: Planar Faults, Bulletin of the Seismological Society of America, 101(5), 2296-2307.

Dunham, E. M., Belanger, D., Cong, L., \& Kozdon, J. E., 2011b. Earthquake ruptures with strongly rateweakening friction and off-fault plasticity, Part 2: Nonplanar faults, Bulletin of the Seismological Society of America, 101(5), 2308-2322.

Duru, K. \& Dunham, E. M., 2016. Dynamic earthquake rupture simulations on nonplanar faults embedded in 3D geometrically complex, heterogeneous elastic solids, Journal of Computational Physics, 305, 185-207.

Fang, Z. \& Dunham, E. M., 2013. Additional shear resistance from fault roughness and stress levels on geometrically complex faults, Journal of Geophysical Research: Solid Earth, 118(7), 3642-3654.

Harbord, C. W., Nielsen, S. B., De Paola, N., \& Holdsworth, R. E., 2017. Earthquake nucleation on rough faults, Geology, 45(10), 931-934.

Haskell, N., 1964. Total energy and energy spectral density of elastic wave radiation from propagating faults, Bulletin of the Seismological Society of America, 54(6), 1811-1841.

Hirakawa, E. \& Ma, S., 2018. Undrained Gouge Plasticity Stabilizes Rupture Dynamics of Rough Faults, Bulletin of the Seismological Society of America.

Huang, Y. \& Gao, H., 2001. Intersonic Crack PropagationPart I: The Fundamental Solution, Journal of Applied Mechanics, 68(2), 169.

Klinger, Y., 2005. High-Resolution Satellite Imagery Mapping of the Surface Rupture and Slip Distribution of the Mw 7.8, 14 November 2001 Kokoxili Earthquake, Kunlun Fault, Northern Tibet, China, Bulletin of the Seismological Society of America, 95(5), 1970-1987.

Klinger, Y., 2010. Relation between continental strike-slip earthquake segmentation and thickness of the crust, Journal of Geophysical Research, 115(B7), B07306.

Klinger, Y., Etchebes, M., Tapponnier, P., \& Narteau, C., 2011. Characteristic slip for five great earthquakes along the Fuyun fault in China, Nature Geoscience, 4(6), 389-392.

Klinger, Y., Okubo, K., Vallage, A., Champenois, J., Delorme, A., Rougier, E., Lei, Z., Knight, E. E., Munjiza, A., Satriano, C., Baize, S., Langridge, R., \& Bhat, H. S., 2018. Earthquake damage patterns resolve complex rupture processes, Geophysical Research Letters.

Kozdon, J. E., Dunham, E. M., \& Nordström, J., 2012. Interaction of Waves with Frictional Interfaces Using Summation-by-Parts Difference Operators: Weak Enforcement of Nonlinear Boundary Conditions, Journal of Scientific Computing, 50(2), 341-367. 
Kozdon, J. E., Dunham, E. M., \& Nordström, J., 2013. Simulation of dynamic earthquake ruptures in complex geometries using high-order finite difference methods, Journal of Scientific Computing, 55(1), 92-124.

Leprince, S., Barbot, S., Ayoub, F., \& Avouac, J.-P., 2007. Automatic and Precise Orthorectification, Coregistration, and Subpixel Correlation of Satellite Images, Application to Ground Deformation Measurements, IEEE Transactions on Geoscience and Remote Sensing, 45(6), 1529-1558.

Manighetti, I., Campillo, M., Sammis, C., Mai, P., \& King, G., 2005. Evidence for self-similar, triangular slip distributions on earthquakes: Implications for earthquake and fault mechanics, Journal of Geophysical Research, 110(B5), B05302.

Milliner, C. W. D., Dolan, J. F., Hollingsworth, J., Leprince, S., Ayoub, F., \& Sammis, C. G., 2015. Quantifying near-field and off-fault deformation patterns of the $1992 \mathrm{M}$ w 7.3 Landers earthquake, Geochemistry, Geophysics, Geosystems, 16(5), 1577-1598.

Milliner, C. W. D., Sammis, C., Allam, A. A., Dolan, J. F., Hollingsworth, J., Leprince, S., \& Ayoub, F., 2016. Resolving Fine-Scale Heterogeneity of Co-seismic Slip and the Relation to Fault Structure, Scientific Reports, 6(1), 27201.

Ozawa, S. W., Hatano, T., \& Kame, N., 2019. Longer Migration and Spontaneous Decay of Aseismic Slip Pulse Caused by Fault Roughness, Geophysical Research Letters, 46(2), 636-643.

Power, W. L. \& Tullis, T. E., 1991. Euclidean and fractal models for the description of rock surface roughness, Journal of Geophysical Research, 96(B1), 415-424.

Power, W. L. \& Tullis, T. E., 1995. A review of the fractal character of natural fault surfaces with implications for friction and the evolution of fault zones, in Fractals in the Earth Sciences, pp. 89-105, Springer.

Renard, F., Voisin, C., Marsan, D., \& Schmittbuhl, J., 2006. High resolution 3D laser scanner measurements of a strike-slip fault quantify its morphological anisotropy at all scales, Geophysical Research Letters, 33(4), L04305.

Rosu, A.-M., Pierrot-Deseilligny, M., Delorme, A., Binet, R., \& Klinger, Y., 2015. Measurement of ground displacement from optical satellite image correlation using the free open-source software MicMac, ISPRS Journal of Photogrammetry and Remote Sensing, 100, 48-59.

Sagy, A. \& Brodsky, E. E., 2009. Geometric and rheological asperities in an exposed fault zone, Journal of Geophysical Research, 114(B2).

Sagy, A., Brodsky, E. E., \& Axen, G. J., 2007. Evolution of fault-surface roughness with slip, Geology, 35(3), 283.

Scholz, C. H., 2019. Mechanics of faulting, in The Mechanics of Earthquakes and Faulting, pp. 97-165, Cambridge University Press.

Shervais, K. A. \& Kirkpatrick, J. D., 2016. Smoothing and re-roughening processes: The geometric evolution of a single fault zone, Journal of Structural Geology, 91, 130-143.

Shi, Z. \& Day, S. M., 2013. Rupture dynamics and ground motion from 3-D rough-fault simulations, Journal of Geophysical Research: Solid Earth, 118(3), 1122-1141.

Spudich, P. \& Frazer, L., 1984. Use of ray theory to calculate high-frequency radiation from earthquake sources 
having spatially variable rupture velocity and stress drop, Bulletin of the Seismological Society of America, 74(6), 2061-2082.

Tal, Y. \& Hager, B. H., 2018. The Slip Behavior and Source Parameters for Spontaneous Slip Events on Rough Faults Subjected to Slow Tectonic Loading, Journal of Geophysical Research: Solid Earth, 123(2), 1810-1823.

Tal, Y., Hager, B. H., \& Ampuero, J. P., 2018. The Effects of Fault Roughness on the Earthquake Nucleation Process, Journal of Geophysical Research: Solid Earth, 123(1), 437-456.

Turcotte, D. L. \& Huang, J., 1995. Fractal Distributions in Geology, Scale Invariance, and Deterministic Chaos, in Fractals in the Earth Sciences, pp. 1-40, eds Barton, C. C. \& La Pointe, P. R., Springer US, Boston, MA.

Ulrich, T., Gabriel, A.-A., Ampuero, J.-P., \& Xu, W., 2019. Dynamic viability of the 2016 Mw 7.8 Kaikura earthquake cascade on weak crustal faults, Nature Communications, 10(1), 1213.

Vallage, A., Klinger, Y., Grandin, R., Bhat, H. S., \& Pierrot-Deseilligny, M., 2015. Inelastic surface deformation during the $2013 \mathrm{M}$ w 7.7 Balochistan, Pakistan, earthquake, Geology, p. G37290.1.

Williams, M. C. B., Duru, K., Gabriel, A. A., \& Dunham, E. M., 2018. 3D Dynamic Rupture on Fractally Rough Faults in Random Heterogeneous Media, Abstract S43A-01 presented at 2018 Fall Meeting, AGU, Washington DC, 1014 Dec..

Yao, Q., 2017. Dynamic Modeling of Earthquake Sources on Rough Faults, Ph.D. thesis, University of California, San Diego.

Zheng, G. \& Rice, J. R., 1998. Conditions under which velocity-weakening friction allows a self-healing versus a cracklike mode of rupture, Bulletin of the Seismological Society of America, 88(6), 1466-1483.

Zielke, O., Galis, M., \& Mai, P. M., 2017. Fault roughness and strength heterogeneity control earthquake size and stress drop, Geophysical Research Letters, 44(2), 777-783. 


\section{APPENDIX A: INFLUENCE OF OFF-FAULT MATERIAL PARAMETERS}

We test here whether changes in the degree of plastic dilatancy $\beta$ and Drucker-Prager viscosity $\eta$ in the medium surrounding the fault will affect slip profiles computed from numerical simulations. The plastic dilatancy $\beta$ is defined as the degree of plastic dilatancy, that is, the ratio of volumetric to shear plastic strain. To be consistent with laboratory experiments measuring dilatancy, Dunham et al. (2011a,b) chose $\beta$ as

$$
\beta=\frac{\mu}{2},
$$

where $\mu$ the Drucker-Prager internal friction parameter.

The Drucker-Prager viscosity $\eta$ is chosen here as

$$
\eta=0.1 G t_{c}
$$

with $G$ shear modulus and $t_{c}$ the characteristic time $t_{c}$ in the simulations, controled by the S-wave speed $c_{S}$, such that $t_{c}=R_{0} / c_{S}$. A nonzero $\eta$ is used in the viscoplastic rheology to ensure wellposed numerical simulations that converge with mesh refinement. The model, as defined, has two characteristic timescales: the viscoplastic relaxation time, $\eta / G$, and the characteristic wave transit time across the state-evolution region, $R_{0} / c_{s}$. The latter also characterizes the timescale over which frictional weakening occurs at the rupture front. A dimensionless ratio of those two terms is thus given by:

$$
\xi=(\eta / G) /\left(R_{0} / c_{s}\right)
$$

When $\xi<<1$, then plasticity is important even during the rapid weakening process at the rupture front. Otherwise, the material response around the rupture front is effectively elastic. In the reference catalogue $\xi=0.1$.

Figure A2 presents the evolution of the Hurst coefficients at very short wavelength $(1 / \lambda>$ $1 \mathrm{~km}^{-1}$ ) as a function of background shear stress. The original simulation, used in Fang \& Dunham (2013) and Bruhat et al. (2016) are indicated in black. Colored error-bars correspond to new set of numerical simulations with different material parameters. We test simulations with a lower $\beta$ (half the original value) and $\beta=0$ (no dilatancy). Additionally, we test values of $\xi$ to half and to twice of its original value. Most simulation are run for $\alpha=0.006$, but we also make some tests at different roughness levels. There seems to be no dramatic change between the simulations, especially in order to reach the range covered by the data displayed in Figure 12 . 

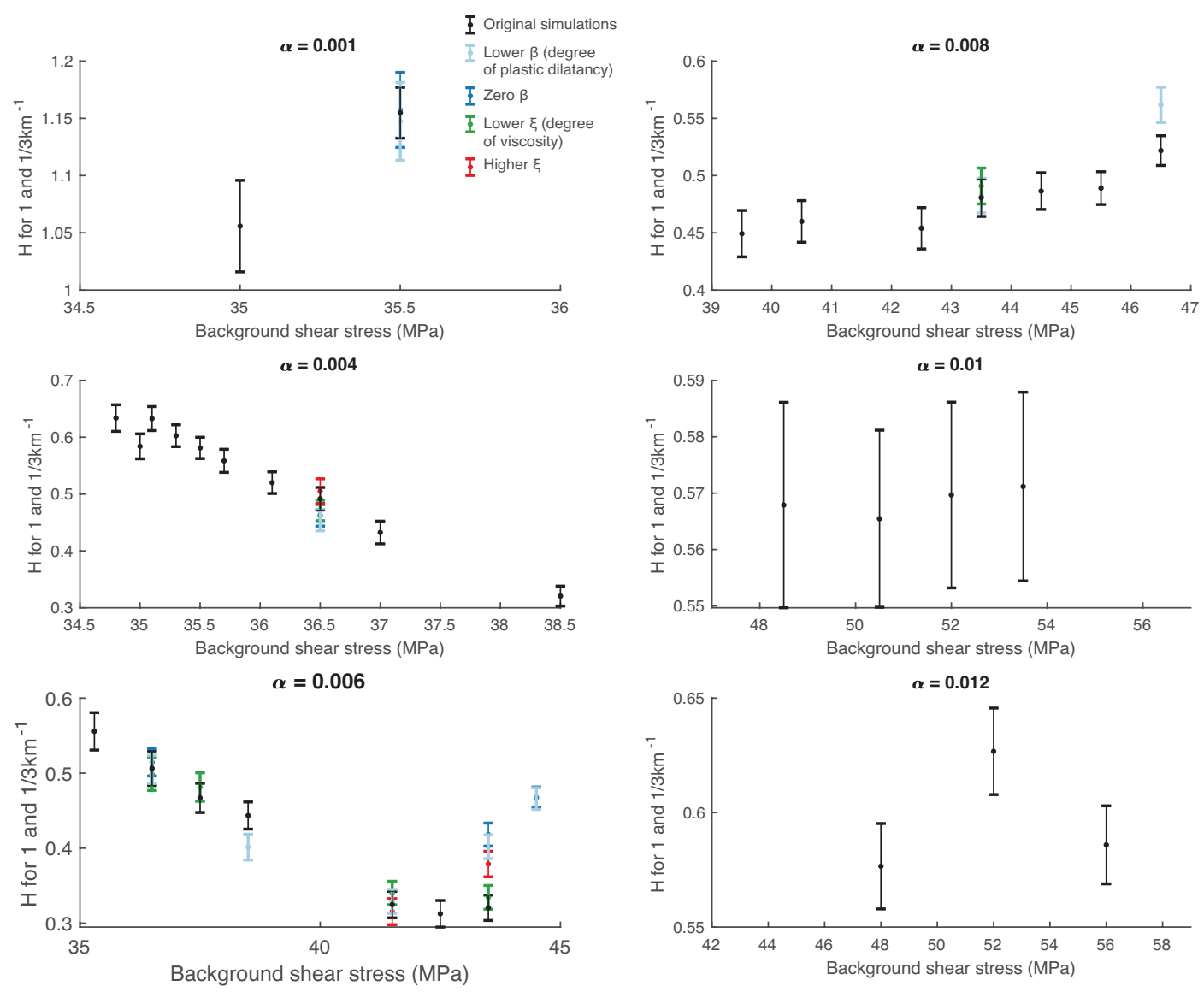

Figure A1. Evolution of Hurst coefficients at very short wavelength $\left(1 / \lambda>1 \mathrm{~km}^{-1}\right)$ as a function of background shear stress. Error bars are for 1- $\sigma$ uncertainties. Black error bars are for simulations with the same parameters for off-fault materials used in Fang \& Dunham (2013) and Bruhat et al. (2016). Colored errors bars correspond to plastic dilatancy or viscosity changes. 
30 Bruhat, et al.
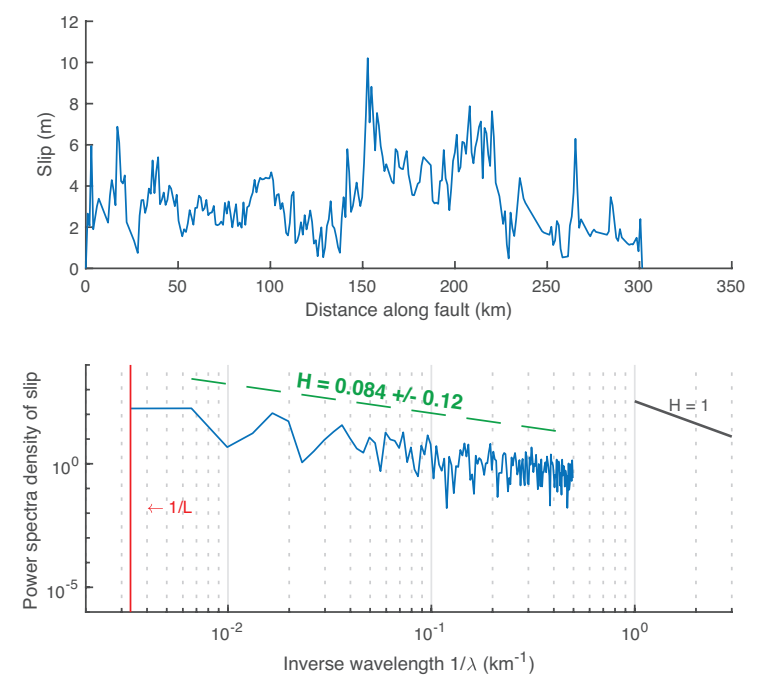

Figure A2. Top) Coseismic slip profile of the $2001 \mathrm{M}_{w} 7.8$ Kunlun earthquake from Klinger (2005). Bottom) Power spectral density of the slip profile, and corresponding Hurst coefficient. Due to a sparser sampling (up to one measurement per km), slip spectra is limited to spatial frequency lower than $0.5 \mathrm{~km}^{-1}$. 
Initial shear/normal stress distribution
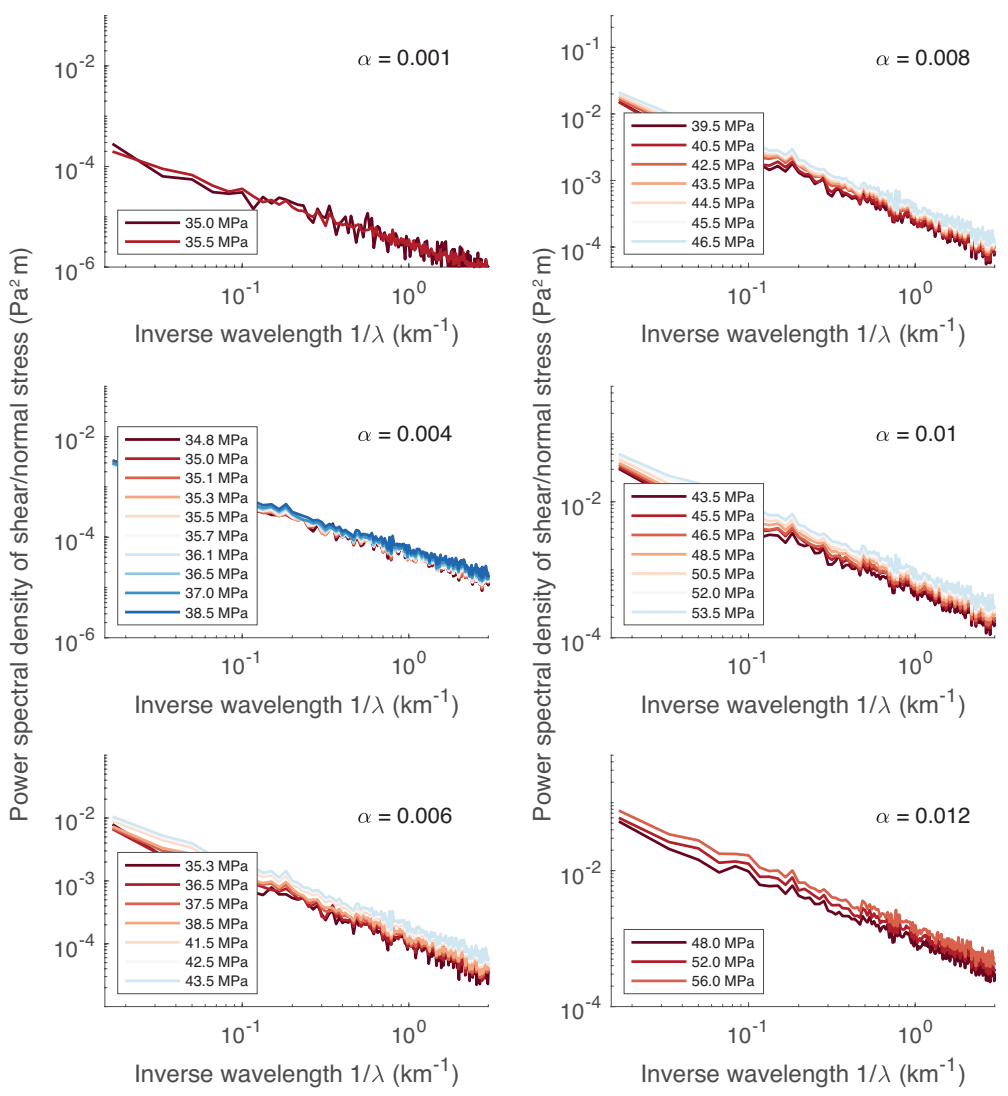

Final shear/normal stress distribution
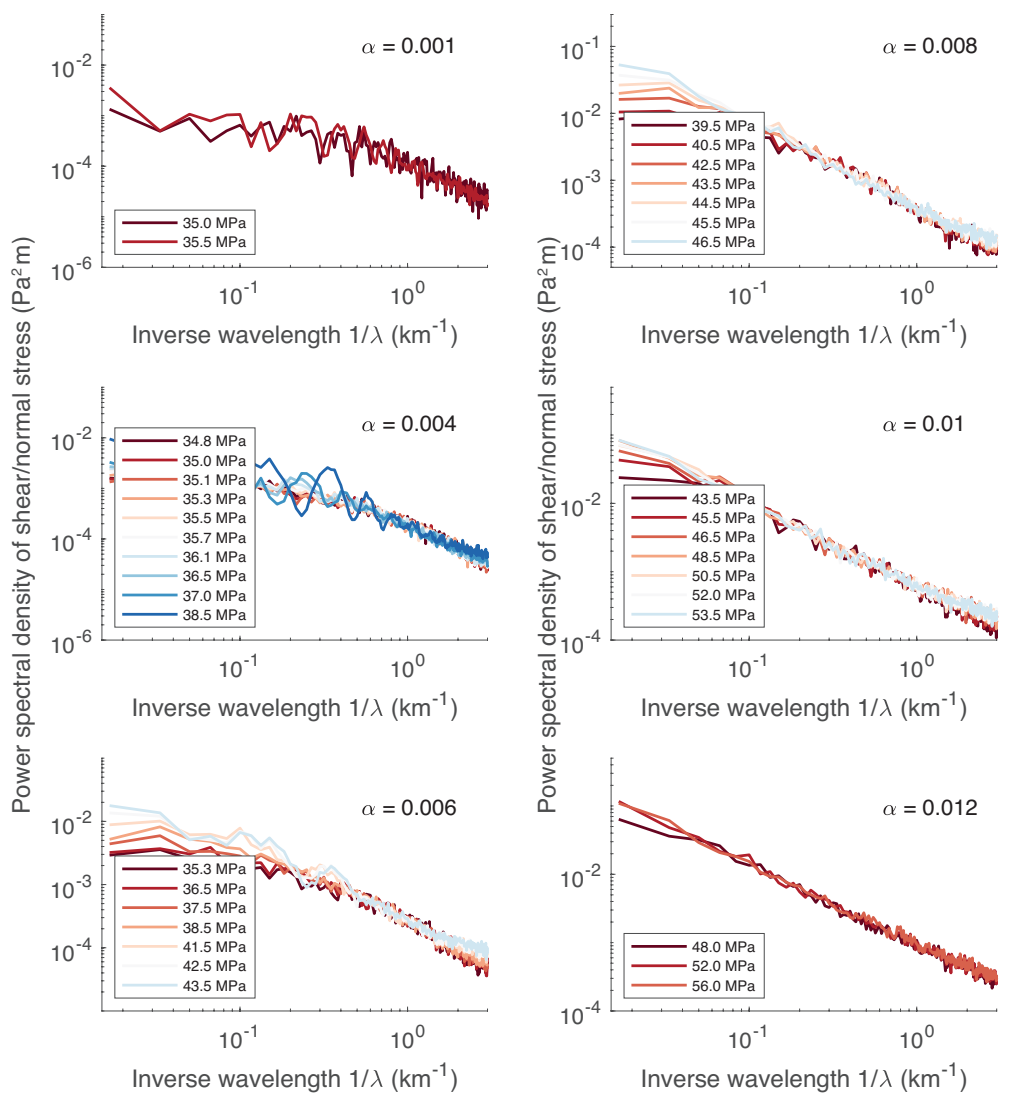

Figure A3. Mean power spectral density of initial and final shear-to-normal stress distributions as a function of the inverse wavelength $1 / \lambda$ and background shear stress, for roughness values from $\alpha=0.001$ to 0.012 . 
Correlation coefficient between the slope $m=d h / d x$ and the gradient of the final slip profile

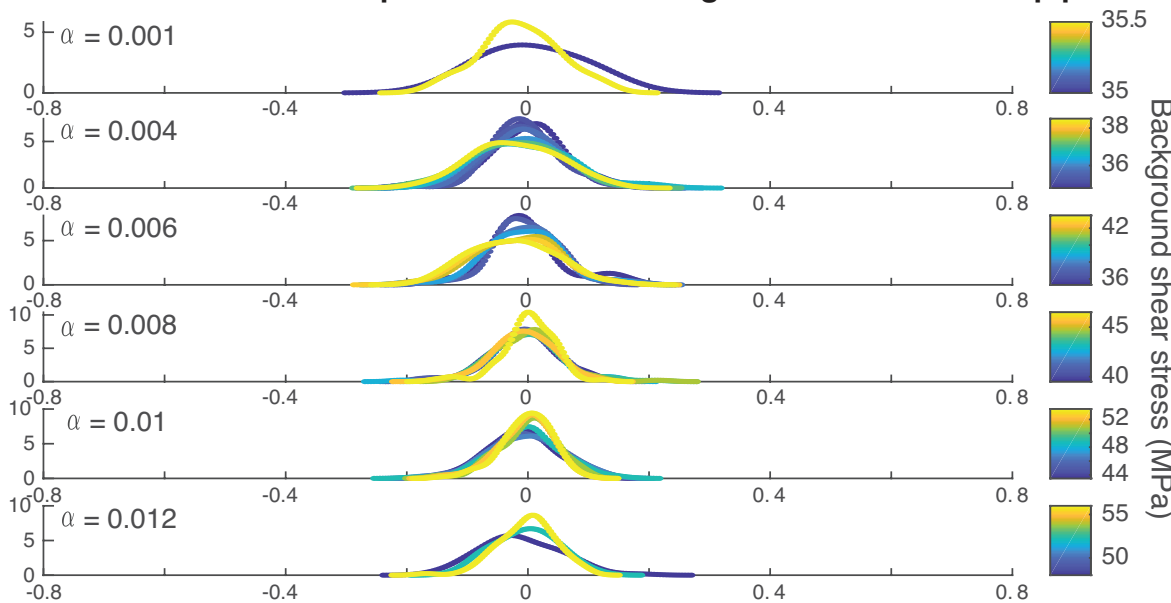

Correlation coefficient between the rupture velocity and the gradient of the final slip profile

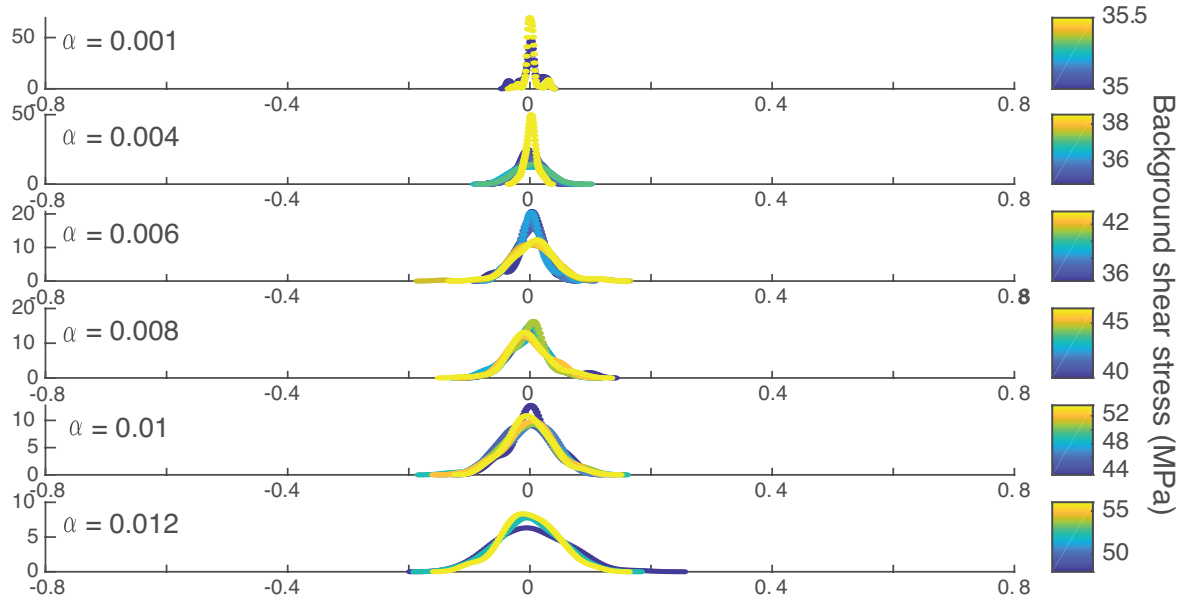

Correlation coefficient between the rupture velocity and the slope $\mathrm{m}=\mathrm{dh} / \mathrm{dx}$

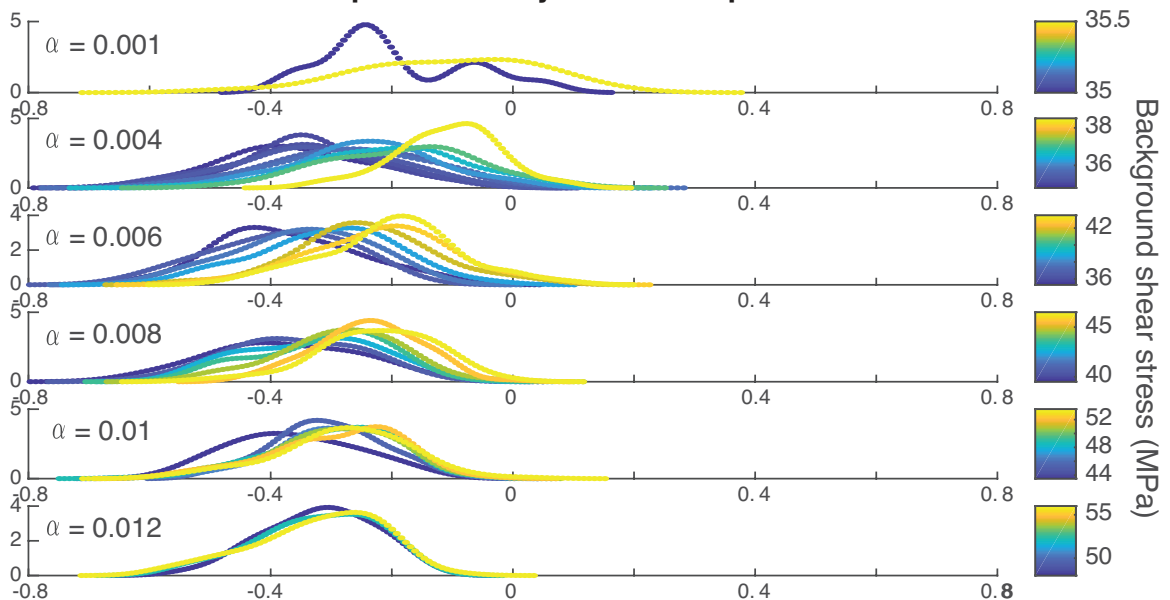

Figure A4. Correlation coefficients between the fault slope $m(x)=\partial y / \partial x$, the gradient of the final slip distribution and the rupture velocity, for all considered roughness $\alpha$ and background shear stress. We only observe the negative correlation between slope and rupture velocity that was already noticed in Fang \& Dunham (2013). 